\title{
An Integrated Approach to Damage Accommodation in Flight Control ${ }^{* \dagger}$
}

\author{
Jovan D. Bošković; Nathan Knoebel ${ }^{\S}$ and Raman K. Mehra, \\ Scientific Systems Company, Inc. \\ Irene Gregory"l \\ NASA Langley Research Center
}

\begin{abstract}
In this paper we present an integrated approach to in-flight damage accommodation in flight control. The approach is based on Multiple Models, Switching and Tuning (MMST), and consists of three steps: - In the first step the main objective is to acquire a realistic aircraft damage model. Modeling of in-flight damage is a highly complex problem since there is a large number of issues that need to be addressed. One of the most important one is that there is strong coupling between structural dynamics, aerodynamics, and flight control. These effects cannot be studied separately due to this coupling.

- Once a realistic damage model is available, in the second step a large number of models corresponding to different damage cases are generated. One possibility is to generate many linear models and interpolate between them to cover a large portion of the flight envelope. Once these models have been generated, we will implement a recently developed Model Set Reduction (MSR) technique. The technique is based on parameterizing damage in terms of uncertain parameters, and uses concepts from robust control theory to arrive at a small number of "centered" models such that the controllers corresponding to these models assure desired stability and robustness properties over a subset in the parametric space. By devising a suitable model placement strategy, the entire parametric set is covered with a relatively small number of models and controllers.

- The third step consists of designing a Multiple Models, Switching and Tuning (MMST) strategy for estimating the current operating regime (damage case) of the aircraft, and switching to the corresponding controller to achieve effective damage accommodation and the desired performance.

In the paper present a comprehensive approach to damage accommodation using Model Set Design, MMST, and Variable Structure compensation for coupling nonlinearities. The approach was evaluated on a model of F/A-18 aircraft dynamics under control effector damage, augmented by nonlinear cross-coupling terms and a structural dynamics model. The proposed approach achieved excellent performance under severe damage effects.
\end{abstract}

\section{Introduction}

There has been an increased interest in addressing flight control problems of aerospace vehicles under adverse conditions, which are defined as failures, damage and/or upset. This interest is motivated by both the desire to ensure safe flight in the presence of adverse conditions and an increased terrorist threat from the man-portable air defense systems (ManPADS). There is a renewed interest in development of effective adaptive reconfigurable control systems that can recover from an adverse event such as damage and reclaim as much vehicle nominal performance as is physically possible. The design of such control systems is a difficult and challenging problem since damage results in both structural and aerodynamic disturbances. For instance, when a wing is damaged, the resulting dynamic disturbance is primarily due to reduced wing stiffness and loss of resistance to flutter. The aircraft can continue to fly after damage only if either

\footnotetext{
${ }^{*}$ Copyright (C) 2007 by Scientific Systems Company, Inc.. Published by the American Institute of Aeronautics and Astronautics, Inc. with permission

${ }^{\dagger}$ This research was supported by NASA Langley Research Center under contract No. NNL07AA02C to Scientific Systems Company.

${ }^{\ddagger}$ Principal Research Engineer \& Intelligent \& Autonomous Control Systems Group Leader, 500 W. Cummings Park, Suite 3000, AIAA Senior Member, jovan@ssci.com

${ }^{\S}$ Research Engineer, 500 W. Cummings Park, Suite 3000, nknoebel@ssci.com

IPresident \& CEO, 500 W. Cummings Park, Suite 3000, AIAA Senior Member, rkm@ssci.com

"Senior Research Engineer, Dynamic Systems and Control Branch, MS 308, Hampton, VA, AIAA Senior Member, irene.m.gregory@nasa.gov
} 
the static stiffness is sufficient to withstand aerodynamic loads, or the damage does not cause loss of flutter controllability and wing destruction. ${ }^{16}$ In addition, wing damage generates asymmetric disturbances that couple longitudinal and lateral dynamics. Hence the general problem of aircraft damage modeling and accommodation is highly complex, and there are only a few results that address this problem under highly restrictive simplifying assumptions.

The problem of controlling a flexible aircraft with significant structural dynamics is highly complex. Initial analyses were performed and solutions to this problem were proposed in the literature. ${ }^{7,10,12-14}$ To address the problem of control effector damage, Wise et $\mathrm{al}^{19}$ assumed that the control effector damage causes the changes in the gain effectiveness matrix, and generates state-dependent disturbances. The scheme estimates the gain effectiveness matrix using a standard adaptive control approach, and the unknown damage-generated nonlinearity using radial basis function neural networks. It was assumed that there is a single nonlinearity due to damage, and that the bounds on the neural network weights are known a priori, which results in the boundedness of the signals in the system. The latter assumption may not be realistic since prior knowledge of the effect of damage is not generally available. In the area of wing damage accommodation, Nguyen and Krishnakumar ${ }^{18}$ developed a vortex-lattice based damage model for various damage configurations, and also added the effect of the loss of symmetry of the damaged aircraft. A control design model was developed through linearization, and a direct adaptive control technique was used to compensate for the damage. The model does not take into account structural model changes and was evaluated for relatively small amounts of damage. The approach by Bošković and Mehra $^{6}$ is based on a restrictive assumption regarding the damage model. The main assumption is that different levels of damage can be modeled as matrix polynomials in the damage paramater that belong from zero to one. No structural effects of damage were taken into account. Similar assumptions were made by Bošković, Bergstrom and Mehra, ${ }^{2}$ where the simultaneous effect of actuator failures and the control effector damage was considered.

In this paper we present an efficient Integrated Damage \& Adaptive Control System (IDACS) that accommodates control effector damage. A block diagram of IDACS is shown in Figure 1. The IDACS is based on the Multiple

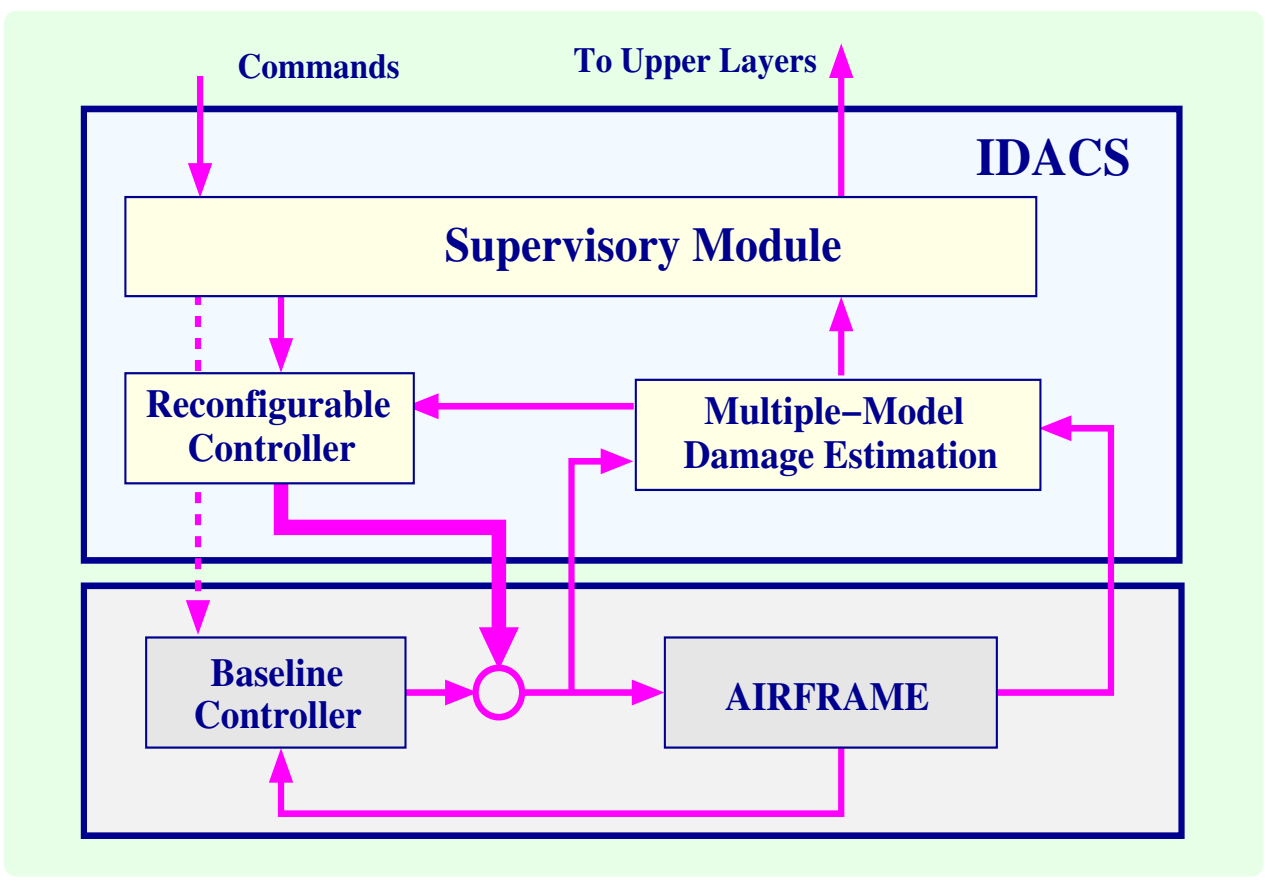

Figure 1. Structure of the Integrated Damage-Adaptive Control System (IDACS).

Models, Switching and Tuning (MMST) technique that has already been applied to the problem of actuator failure accommodation. ${ }^{5,17}$ The current approach is based on developing coupled structural dynamics and aerodynamic models for different damage amounts, deriving a suitable parametrization of the models in order to generate a relatively small number of models covering the entire parametric set, and building corresponding multiple-model estimators. The estimates of unknown nonlinearities can be generated using either neural networks or Variable Structure techniques. If a solution to the wing damage accommodation problem exists, the use of these estimates in the reconfigurable control 
law will assure stabilization of the aircraft in the presence of wing damage. This is an important problem that has not been solved so far, and its solution will contribute to improved flight safety of both commercial and military aircraft.

This research has been funded by the Integrated Resilient Aircraft Control (IRAC) project under NASA's Aviation Safety Program. The goal of the IRAC project is to arrive at a set of validated multidisciplinary integrated aircraft control design tools and techniques for enabling safe flight in the presence of adverse conditions (ex: faults, damage and/or upsets). Major elements of the IRAC project involve integrated modeling of aerodynamics and structures; adaptive control under adverse conditions; and verification and validation of models and control methodologies. Hence, the approach to mitigate the impact of damage on the stability and performance of aircraft proposed in this paper contributes to two of the three areas emphasized in IRAC in an integrated fashion.

To conduct the research under this project we obtained a simple model of wing damage in F/A-18 aircraft form Boeing that simulates control effector damage effects on aircraft dynamics, and augmented it with cross-coupling terms due to assymmetry caused by damage, as well as by a structural model of the TAMU wing. ${ }^{20}$ In the paper present a comprehensive approach to damage accommodation using Model Set Design, MMST, and Variable Structure compensation for coupling nonlinearities. Several issues have been specifically addressed: (i) How to arrive at a simple Model Set Design (MSD) procedure? (ii) How to include the cross coupling terms into post-damage aircraft dynamics, and how to compensate for these terms? and (iii) How to integrate rigid-body and structural dynamics into a meaningful model and design a corresponding MMST controller?

\section{Damage Models and Baseline Control Strategies}

In this section we describe three damage models of increasing complexity that were used under the project. Associated with each of the models is a baseline control strategy used as basis for adaptive and MMST-based control design.

Under this project we obtained a simple linear simulation model of wing damage in F/A-18 aircraft from Boeing. The flight regime is 0.7 Mach and 20,000, and the corresponding $A$ and $B$ matrices are modified to include perturbations due to damage. This model was used as a baseline, and was subsequently augmented by inter-axes coupling effects due to damage, and by a structural model consistent with that derived for the TAMU wing. ${ }^{20}$

The nominal F/A-18 linear model is of the form:

$$
\dot{x}=A_{n} x+B_{n} u
$$

with perturbed states and inputs

$$
\begin{aligned}
& x=[u, q, \theta, \alpha, h, \beta, p, r, \phi, \psi]^{T} \\
& u=\left[\mathrm{LEF}_{l}, \mathrm{LEF}_{r}, \mathrm{TEF}_{l}, \mathrm{TEF}_{r}, \mathrm{AIL}_{l}, \mathrm{AIL}_{r}, \mathrm{STAB}_{l}, \mathrm{STAB}_{r}, \mathrm{RUD}_{l}, \mathrm{RUD}_{r}, \mathrm{PLA}_{l}, \mathrm{PLA}_{r}\right]^{T} .
\end{aligned}
$$

In the case with no damage, the baseline controller is of the form:

$$
u=B_{n}^{\dagger}\left(-A_{n} x+A_{m} x+B_{m} r\right)
$$

where $B_{n}^{\dagger}$ denotes a pseudo-inverse of $B_{n}$.

The damage model includes the nominal system matrices $A_{n}$ and $B_{n}$, plus two additive damage perturbations matrices such that

$$
\dot{x}=\left(A_{n}+\Delta A\left(\theta_{1}\right)\right) x+\left(B_{n}+\Delta B\left(\theta_{2}\right)\right) u
$$

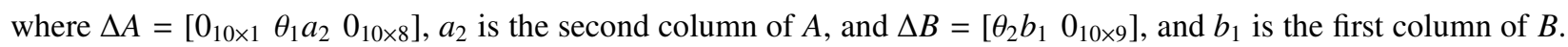
Boeing engineers feel that this model adequately describes small wing damage in F/A-18 aircraft.

We note that the assumed damage perturbations are defined using two uncertain terms, namely $\theta_{1}$ and $\theta_{2}$, where only the columns of the state and input matrices associated with $q$ and $\mathrm{LEF}_{l}$ are affected. For this example it is assumed that all states are measured, actuator dynamics are negligible, and the control objective is to follow the reference model:

$$
\dot{x}_{m}=A_{m} x_{m}+B_{m} r .
$$

In this case, the baseline controller is of the form:

$$
u=\left(B_{n}+\Delta B\left(\hat{\theta}_{2 k}\right)\right)^{\dagger}\left(-\left(A_{n}+\Delta A\left(\hat{\theta}_{1 k}\right)\right) x+A_{m} x+B_{m} r\right) .
$$


To take into account coupling between longitudinal and lateral dynamics due to the loss of symmetry in the case of damage, possible coupling nonlinearities are introduced into the model of F/A-18 dynamics. The resulting additional terms are combined into a single vector, namely $\xi$ as

$$
\dot{x}=\left(A_{n}+\Delta A\left(\theta_{1}\right)\right) x+\left(B_{n}+\Delta B\left(\theta_{2}\right)\right) u+\xi
$$

where

$$
\xi=\left[\begin{array}{c}
0 \\
-9 \alpha-22.5 \beta-4.5 p-9 q-18 r-4.5 p q-4.5 p r-9 q r \\
0 \\
0 \\
0 \\
0 \\
-22.5 \alpha-13.5 \beta-4.5 p-13.5 q-36 r-9 p q-9 p r-4.5 q r \\
-4.5 \alpha-22.5 \beta-13.5 p-22.5 q-9 r-18 p q-18 p r-4.5 q r \\
0 \\
0
\end{array}\right] .
$$

The effect of $\xi$ can be addressed using variable structure or sliding mode control for the compensation of the effect of nonlinearities. To accomplish this, $\hat{\xi}_{k}$ is included in the current model, with $k$ corresponding to the current model, such that

$$
\dot{\hat{x}}_{i}=\left(A_{n}+\Delta A\left(\hat{\theta}_{1 k}\right)\right) x+\left(B_{m}+\Delta B\left(\hat{\theta}_{2 k}\right)\right) u+\hat{\xi}_{k}-\lambda \hat{e}_{k} .
$$

Note that the vector estimating the nonlinearities $\hat{\xi}_{k}$ is adjusted using

$$
\hat{\xi}_{k}=-E \bar{\xi}
$$

where $E=\operatorname{diag}\left[\left(\operatorname{sign}\left(\hat{e}_{k}\right)\right)\right]$ and

$$
\bar{\xi}=\left[\begin{array}{c}
0 \\
22.5(|\alpha|+|\beta|+|p|+|q|+|r|)^{2} \\
0 \\
0 \\
0 \\
0 \\
36(|\alpha|+|\beta|+|p|+|q|+|r|)^{2} \\
22.5(|\alpha|+|\beta|+|p|+|q|+|r|)^{2} \\
0 \\
0
\end{array}\right]
$$

The estimate of the nonlinearities is then added to the control law to give

$$
u=\left(B_{n}+\Delta B\left(\hat{\theta}_{2 k}\right)\right)^{\dagger}\left(-\left(A_{n}+\Delta A\left(\hat{\theta}_{1 k}\right)\right) x-\hat{\xi}_{k}+A_{m} x+B_{m} r\right) .
$$

As a next step in our evaluation of the suitability of the MMST approach to handle structural damage, we integrated the F/A-18 damage model with that of the TAMU wing, ${ }^{20}$ and assumed coupling between these subsystems as well as parameter changes due to damage. The criterion for choosing the coupling terms was that the system quickly becomes unstable in the case of damage.

The augmented model is of the form:

$$
\begin{aligned}
& \dot{x}_{S}=A_{S}\left(\theta_{1}\right) x_{S}+B_{S}\left(\theta_{2}\right) u+A_{R S}\left(\theta_{1}\right) x_{R} \\
& \dot{x}_{R}=A_{R}\left(\theta_{1}\right) x_{R}+B_{R}\left(\theta_{2}\right) u+A_{S R}\left(\theta_{1}\right) x_{S}+\xi
\end{aligned}
$$

where $x_{R} \in \mathbb{R}^{n}$ is the vector of rigid body states, $x_{S} \in \mathbb{R}^{l}$ is the vector of pitch-and-plunge states, and $u \in \mathbb{R}^{m}$ is the control input vector. $A_{S}, B_{S}, A_{R S}, A_{R}, B_{R}$, and $A_{S R}$ are matrices of appropriate dimensions. It is assumed that the above system is completely controllable and observable, which is a realistic assumption.

$$
4 \text { of } 25
$$


The control objective is to design a control input $u(t)$ so that $x_{R}(t)$ follows a rigid-body reference model, while the pitch-and-plunge states are stabilized around zero, i.e. $\lim _{t \rightarrow \infty} x_{S}(t)=0$ despite the large changes in $\theta$ and $\xi$.

We note that the baseline controller for this case is similar to the previous one, except that it is now designed for an extended state space, consisting of both rigid-body and structural states. A solution to this problem in the case of large uncertainties in $\theta$ and $x i$ is proposed in this paper, and consists of model set design and reduction, and implementation of a MMST scheme for the augmented damage model.

\section{A Model Set Design Approach}

In this section the focus is on the model set design for multiple model control.

Let a parametric set be defined as: $\mathcal{S}_{\theta}=\left\{\theta:\left(\theta_{i}\right)_{\min } \leq \theta_{i} \leq\left(\theta_{i}\right)_{\max }\right.$, for $\left.i=1,2, \ldots, N\right\}$ where $N$ is the number of uncertain parameters. Then the multiple model control technique involves placing a finite number of models within this set, designing corresponding observers, and on-line selection of a single controller from a set of controllers also corresponding to the predetermined models and observers. This selection is usually based upon some comparison metric to determine which observer is closest in some sense to the true system from observations made. A control strategy is then applied given the current observer selection.

The choice of the control strategy is relevant for model set design based on performance specifications for a closedloop system. This will be discussed in more detail below.

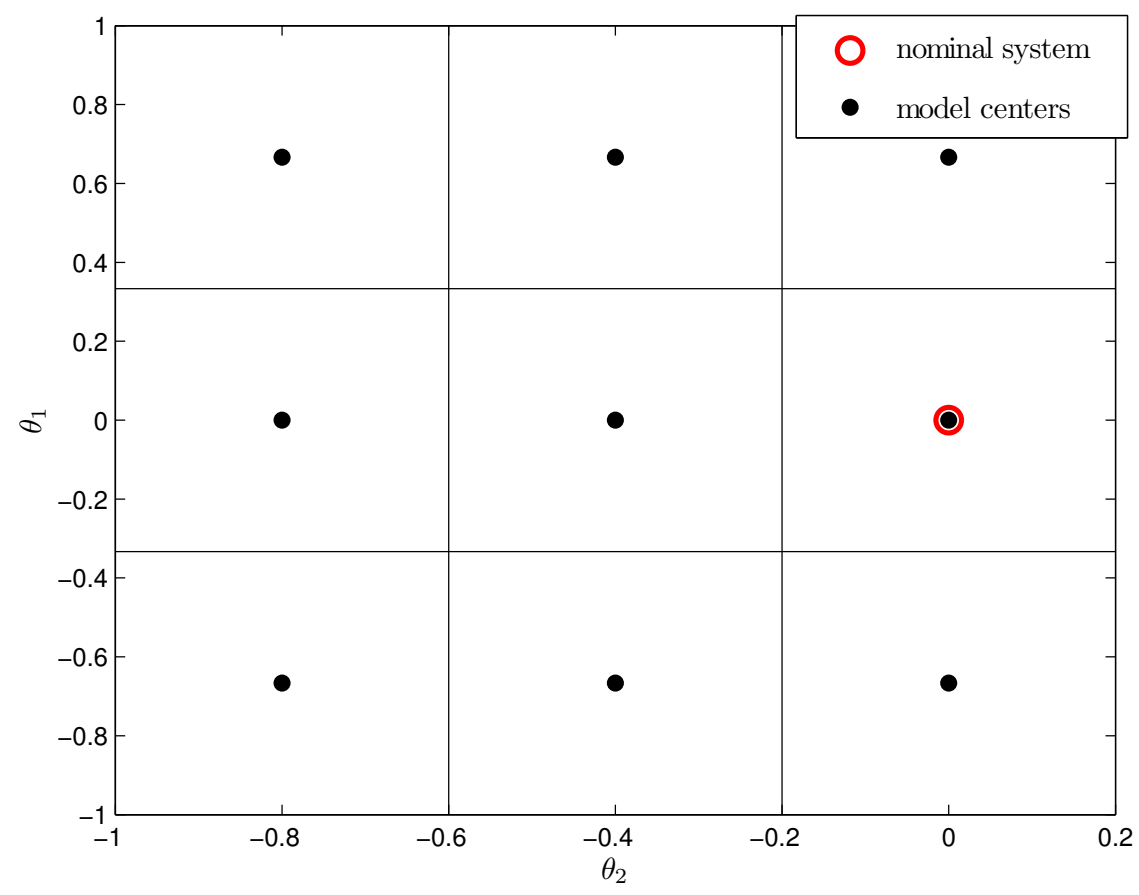

Figure 2. Example model set design with equally spaced models and the same number of model divisions for each uncertain parameter.

For the F/A-18 application, it is assumed that the uncertain set is defined as $\mathcal{S}_{\theta}=\left\{\left(\theta_{1}, \theta_{2}\right):-1 \leq \theta_{1} \leq 1 ;-1 \leq\right.$ $\left.\theta_{2} \leq 0\right\}$, where a single model can be specified by a point $\theta=\left[\theta_{1}, \theta_{2}\right]^{T} \in \mathcal{S}_{\theta}$. One possibility for the model set design is based on equally spaced models with the same number of model divisions for each uncertain parameter. An example of this approach is shown in Figure 2. For control the inverse dynamics strategy is chosen for this application, yielding

$$
u=\left(B_{n}+\Delta B\left(\hat{\theta}_{2 k}\right)\right)^{-1}\left(-\left(A_{n}+\Delta A\left(\hat{\theta}_{1 k}\right)\right) x+A_{m} x+B_{m} r\right)
$$

where $k$ is the model that is currently selected, and $\hat{\theta}_{1 k}$ and $\hat{\theta}_{2 k}$ are the corresponding parameters of that model. This 
strategy results in the following closed-loop system:

$$
\begin{aligned}
\dot{x}= & {\left[A_{n}+\Delta A\left(\theta_{1}\right)+\left(B_{n}+\Delta B\left(\theta_{2}\right)\right)\left(B_{n}+\Delta B\left(\hat{\theta}_{2 k}\right)\right)^{-1}\left(-\left(A_{n}+\Delta A\left(\hat{\theta}_{1 k}\right)\right)+A_{m}\right)\right] x } \\
& +\left(B_{n}+\Delta B\left(\theta_{2 k}\right)\right)\left(B_{n}+\Delta B\left(\hat{\theta}_{2 k}\right)\right)^{-1} B_{m} r .
\end{aligned}
$$

Proposed Model Set Design Approach: Our model set design approach is based on equally spaced models within the parametric set. We first define the "centered" models. For instance, if the model set has a single parameter such that $\theta \in[0,1]$, let $N$ be the number of models that will be used to cover this interval. Then the length of each subinterval will be $1 / N$. Let us assume that model centers should be also placed at interval boundaries. In that case the total number of models is $N+1$, and the location of the "centered" models will be at $0,1 / N, 2 / N, \ldots 1-1 / N, 1$. If the models do not need to be placed at the interval boundaries, then there will be exactly $N$ models located at: $1 / N / 2,3 / N / 2, \ldots 1-1 / N / 2$.

The next issue to be addressed is how to choose $N$. One possibility is to make that choice based on some stability and/or performance criterion. We note that a given $N$ defines a set of equally spaced centered models. For the 2 parameter application it can be seen that for model $j$, the set of values that satisfy

$$
\left\|\theta-\theta_{j}\right\|_{2} \leq\left\|\theta-\theta_{i}\right\|_{2} \quad \text { for } i \neq j
$$

defines $\mathcal{S}_{\theta_{j}}$, a rectangular subset of $\mathcal{S}_{\theta}$ for which model $j$ is the closest. Observe that the controller for model $j$ is defined by (13) where $k=j$ yielding the closed-loop system in (14). To evaluate the case when $j$ is the selected model and $\theta \in \mathcal{S}_{\theta_{j}}$ we consider the cases when the true plant is at the corners of $\mathcal{S}_{\theta_{j}}$, and evaluate if the resulting closed-loop systems satisfy performance specifications. For instance, one possible criterion is that the closed-loop eigenvalues must be within a specified euclidean distance from the desired eigenvalues of the reference model. By evaluating the corners of the models, one can ensure that, if the correct model is selected and the controller based on the model center is chosen, the closed-loop system will be guaranteed to be close to the reference model in the sense of eigenvalue location. Figure 3 illustrates this concept.

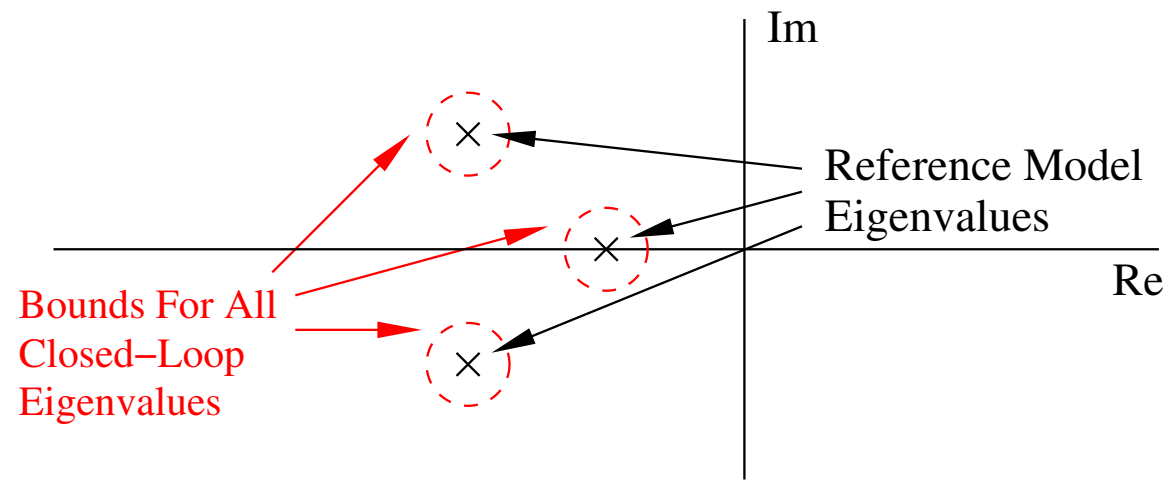

Figure 3. Evaluation criteria for the model set design: closed-loop poles need to be located within red circles.

A simple algorithm for ensuring the least number of models where all of the models meet the specifications described above is as follows:

\section{Algorithm:}

1. Set maximum number of model divisions for each parameter

2. Divide the space into even models

3. Evaluate the closed-loop system with the control parameters at the model centers and the true system parameters at the model corners

- If all models pass, then reduce the number of model divisions for each parameter and return to step 2

- If any model fails, then increase the number of model divisions for each parameter to the value that passed in the previous iteration, and quit. 


\section{Multiple-Model-based Control Design}

In this section we first give a general description of the Multiple Models, Switching and Tuning (MMST) technique, followed by a description of several different switching strategies that improve the overall system robustness.

\section{A. Multiple Models, Switching and Tuning (MMST)}

The concept of MMST is based on the idea of describing the dynamics of the system using different dynamic models for different operating regimes; such models are close in some sense to the dynamics of the system in different operating regimes, and the corresponding observers are consequently referred to as the identification models. The basic idea is to set up such identification models and corresponding controllers in parallel, Figure 4, and to devise a suitable strategy for switching among the controllers to achieve the desired control objective. While the plant is being controlled using one of these controllers, the identification models are run in parallel to generate some measure of the corresponding identification errors and to ultimately determin which observer is, in some sense, closest to the current operating regime of the plant. Once such an observer is found, the switching mechanism switches to (or stays at) the corresponding controller, where the switching interval is a parameter chosen by the designer.

The main feature of this approach is that in LTI systems, if one of the models is adaptive where estimated parameters are limited only by the bounds of the uncertainty space, the approach results in a stable overall system in which asymptotic convergence of the output error to zero is guaranteed under relatively mild conditions. In addition, if adaptive control is used and the controller parameters are adjusted using adaptive algorithms with projection, the overall system is robust to different perturbations, including bounded external disturbances, time variations of plant parameters, and some classes of unmodeled dynamics. As also shown through extensive simulations, the performance of the overall switching system can be dramatically improved as compared to that achieved using a single adaptive controller.

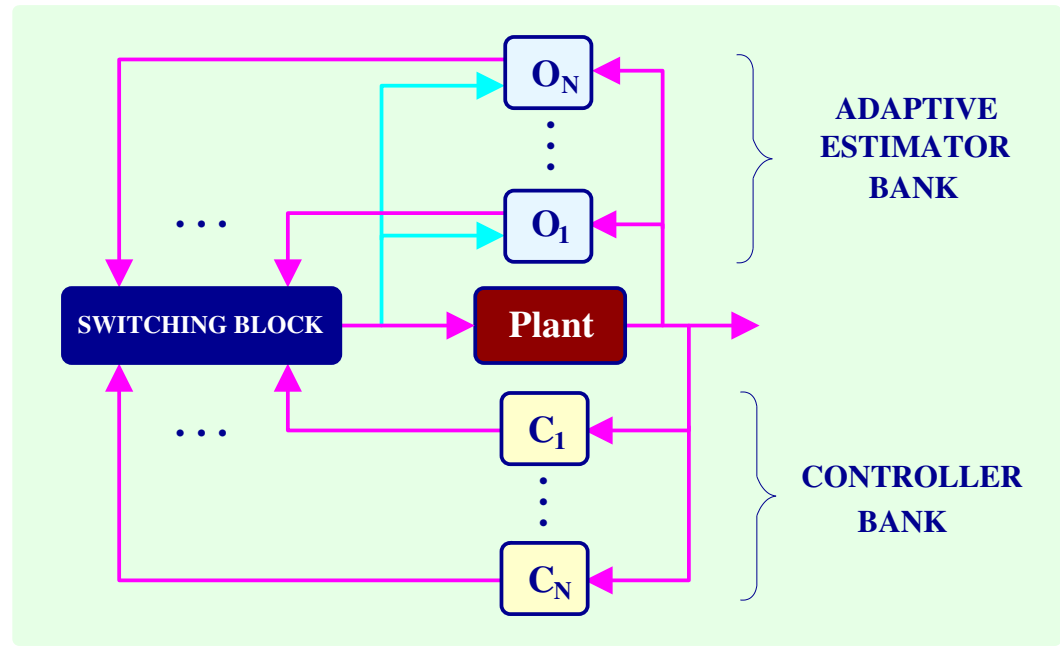

Figure 4. Structure of the Multiple Model-Based Controller: Outputs of the parallel observers $O_{1}, O_{2}, \ldots O_{N}$ are used to find that closest in some sense to the current plant dynamics, and switch to the corresponding controller.

In the context of reconfigurable control design in the presence of parametric uncertainties and/or sensor, actuator and structural failures, the identification models (observers) $O_{1}, \ldots, O_{N}$ from Figure 4 correspond to different regions in the parameter space characterizing different types of failures, while $C_{1}, \ldots, C_{N}$ denote the corresponding controllers.

In the context of damage accommodation, we use the MMST approach with a model that corresponds to the nominal (no-damage) case, and damage models that cover the entire parametric set. The best estimate at every instant, as selected by the MMST subsystem, is used in the control law.

\section{B. Switching Strategies}

Switching from one controller to another within the MMST framework is based on the comparison of estimation errors representing a difference between the outputs of on-line observers and observations of the the true system. For the 
F/A-18 model the observers differ by the values of $\hat{\theta}_{1 i}$ and $\hat{\theta}_{2 i}$, and are of the form:

$$
\dot{\hat{x}}_{i}=\left(A_{n}+\Delta A\left(\hat{\theta}_{1 i}\right)\right) x+\left(B_{m}+\Delta B\left(\hat{\theta}_{2 i}\right)\right) u-\lambda \hat{e}_{i} \quad \text { for } i=1,2, \ldots n
$$

where $n$ is the number of models, and $\hat{e}_{i}=\left(\hat{x}_{i}-x\right)$.

The metric employed for model comparison is the integral of the squared observer error over a finite time period. Corresponding performance indices are of the form:

$$
I_{i}(t)=\int_{t-\Delta t}^{t} \hat{e}_{i}^{T}(\tau) \hat{e}_{i}(\tau) d \tau \quad \text { for } i=1,2, \ldots n,
$$

and the current model selection index satisfies

$$
i^{*}=\arg \min _{i} I_{i}(t), \quad \text { for } i=1,2, \ldots n .
$$

One disadvantage of the decision making based on this criterion is that, in the case of all fixed observers where all model parameter estimates are fixed at the model centers, switching to a wrong observer can occur. To gain more insight into this problem, the error model using equations (1) and (16), is written as:

$$
\dot{\hat{e}}_{i}=\Omega \phi_{i}-\lambda \hat{e}_{i}
$$

where $\phi_{i}=\left[\hat{\theta}_{1 i}-\theta_{1}, \hat{\theta}_{2 i}-\theta_{2}\right]^{T}$ and $\Omega=\left[\begin{array}{ll}a_{2} q & b_{1} \delta_{L E F}\end{array}\right]$ yielding

$$
\begin{aligned}
\hat{e}_{i} & =\Omega_{F} \phi_{i}+\xi_{i} \\
\dot{\Omega}_{F} & =-\lambda \Omega_{F}+\Omega \\
\dot{\xi}_{i} & =-\lambda \xi_{i}-\Omega_{F} \dot{\phi}_{i}
\end{aligned}
$$

where if $\phi_{i}$ is constant $\xi_{i}=0$, while if $\phi_{i}$ is not constant but changes only infrequently $\xi_{i}$ is an exponentially decaying signal whose decay rate is determined by the observer gain $\lambda$. Now assuming that $\xi_{i} \approx 0$ the performance indices are rewritten as:

$$
I_{i}(t)=\int_{t-\Delta t}^{t} \hat{e}_{i}^{T}(\tau) \hat{e}_{i}(\tau) d \tau=\phi_{i}^{T}\left(\int_{t-\Delta t}^{t} \Omega_{F}^{T}(\tau) \Omega_{F}(\tau) d \tau\right) \phi_{i} .
$$

From this point one can see that in order to guarantee that the system will switch to model closest to the current plant dynamics in the euclidean sense (i.e. $i^{*}=\arg \min _{i}\left\|\phi_{i}\right\|_{2}$ ), the singular values of the matrix

$$
\int_{t-\Delta t}^{t} \Omega_{F}^{T}(\tau) \Omega_{F}(\tau) d \tau
$$

must be equal. Geometrically in the 2 parameter application the level sets of

$$
\int_{t-\Delta t}^{t} \hat{e}_{i}^{T}(\tau) \hat{e}_{i}(\tau) d \tau
$$

in the two dimensional parameter space defined by $\mathcal{S}_{\theta}$ must be circular in order to guarantee that model with the smallest parametric error will be selected. From (23) it can be seen that this coinsides with the singular values of (24) being equal. Since this is not guaranteed, switching to the wrong model can occur. From simulations and testing it has been found that, to reduce this undesired phenomena in multiple model selection, several techniques can be applied.

Making the current model adaptive and all other models fixed: This method of all tested appears to have the greatest influence on undesired switching. For the current model estimates, the parameters are adjusted using the adaptation law

$$
\dot{\hat{\theta}}_{i}=\operatorname{Proj}_{\mathcal{S}_{\theta_{i}}}\left[-\Gamma \Omega^{T} \hat{e}_{i}\right]
$$

where $\Gamma=\operatorname{diag}\left(\left[\gamma_{1}, \gamma_{2}\right]\right), \gamma_{1}, \gamma_{2}>0$. Also note that $\operatorname{Proj}_{\mathcal{S}_{\theta_{i}}}[(\cdot)]$ is the projection operator with $\mathcal{S}_{\theta_{i}}=\left\{\theta_{1 i}, \theta_{2 i}\right): \underline{\theta}_{1 i} \leq$ $\left.\theta_{1 i} \leq \bar{\theta}_{1 i} ; \underline{\theta}_{2 i} \leq \theta_{2 i} \leq \bar{\theta}_{2 i}\right\}$, where $\underline{\theta}_{j i}$ and $\bar{\theta}_{j i}$ for $j=1,2$ denote the lower and upper parameter boundaries respectively for an individual model $i$. This method reduces incorrect switching as follows. Through adaptation, the observer that is currently selected is able to outperform the other fixed observers by locating local parameters within the current model 
boundaries. This typically occurs until the observer of a model that is truly closer to the actual system (i.e. in the sense of parameter location) results in a better performance index.

Bias the current model: In this case the performance indices are modified as follows:

$$
I_{i}(t)=\left\{\begin{array}{ll}
\int_{t-\Delta t}^{t} \hat{e}_{i}^{T}(\tau) \hat{e}_{i}(\tau) d \tau-\delta & \text { if } i=k \\
\int_{t-\Delta t}^{t} \hat{e}_{i}^{T}(\tau) \hat{e}_{i}(\tau) d \tau & \text { otherwise }
\end{array}\right\}
$$

where $\delta>0$ is the bias, $I_{i}$ is the comparison metric, and $k$ is the current model. With this technique the selection will favor the current model unless the current model error is sufficiently large.

Decrease the selection frequency with adaptation of the current model: Selection frequency has also been found to reduce unnecessary model switching when the current model is adaptive and other models are fixed. By increasing the time between model selection it is assumed that the adaptive model has more opportunity to adapt and outperform other incorrect fixed models.

\section{Simulations}

Simulations of the multiple model design and control strategies described is given below.

The simulation scenario is the F/A-18 performing a pitch doublet maneuver. It is assumed that the damage parameters change from $\theta=[0,0]^{T}$ to $\theta=[0.4687,-0.4653]^{T}$ at 3 seconds. For model set design, the closed loop eigenvalues are constrained to be within 0.2 of the reference model eigenvalues. This leads to a set of equally spaced models with five divisions for each parameter, or the total of 25 models. First the results for all fixed models with an observer gain of $\lambda=20$ and integration time of $\Delta t=0.2$ seconds are given.

\section{A. Damage model without augmentation}

This case served primarily for choosing the most suitable MMST strategy and tuning of the MMST controller. The response of the system with the MMST controller is shown in Figures 5 and 6. It can be seen that with fixed models the system states are well behaved but incorrect switching occurs.

This however, is shown to be remedied in the following simulation where switching improvement techniques described earlier are employed. Under the same scenario, the current model is made adaptive, while all others are fixed. Adaptation gains are set to $\gamma_{1}=70$ and $\gamma_{2}=500$, the observer gain is set to $\lambda=20$, and $\Delta t=0.2$. Also, biasing is applied with

$$
\delta(t)=0.2 \int_{t-\Delta t}^{t} \hat{e}_{i}^{T}(\tau) \hat{e}_{i}(\tau) d \tau
$$

and the switching frequency is reduced to wait 0.2 seconds between model selections. Results for this simulation are given in Figures 7 and 8. The longitudinal states are shown to track the reference model accurately. It is also seen that the switching is well behaved, due to the use of the previously described techniques that prevent switching to a wrong model.

\section{B. Damage model with inter-axes coupling}

Without compensation, the addition of these nonlinear terms to the F/A-18 dynamics results in instability as shown in Figure 9. In this simulation the same MMST controller described above (with the current model adaptive, biasing, and decreased switching frequency) is applied to the damage scenario where, after 3 seconds, $\theta=[0,0]^{T}$ changes to $\theta=[0.4687,-0.4653]^{T}$, and $\xi$ is introduced.

With the addition of the variable structure term to the previous multiple model controller, the same highly nonlinear system can be stabilized, and can accurately track the reference system. This is shown in Figure 10, which shows the longitudinal states of the nonlinear system throughout the doublet command. Figure 12 shows the actual nonlinearities and the variable structure terms used for cancellation. The damage parameters of the system can also be seen in Figure 11 where the model switching and parameter estimates are well behaved with the addition of $\hat{\xi}_{k}$ to the observers and controllers. 

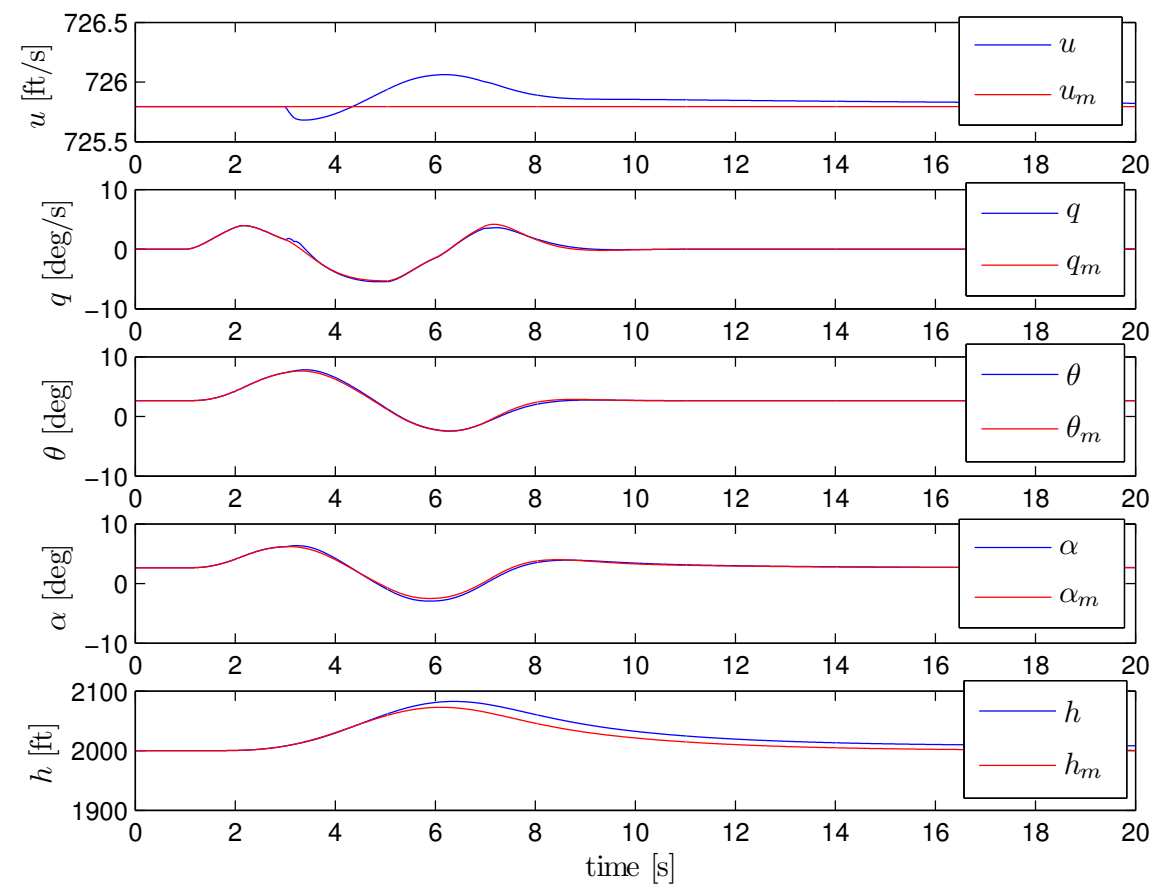

Figure 5. MMST with Fixed Models: Response of the longitudinal states under damage at $t=3$ seconds.

\section{MMST-based Control of an Aero-Servo-Elastic Model}

We first simulated the case when the system is controlled using a reduced-order controller, i.e. the controller that does not take into account pitch-and-plunge states. Figure 13-14(b) show the resulting response. It is seen that the longitudinal state errors are large, while the pitch-and-plunge states are highly oscillatory. The response of the parameter estimates is also seen to be highly oscillatory, while the switching pattern exhibits a similar response.

The next simulation is of the case when the system is controlled using a full-order controller, i.e. the controller that takes into account pitch-and-plunge states. Figure 16-17(b) show the resulting response. It is seen that the longitudinal state errors are much smaller than in the previous case, while the oscillations of the pitch-and-plunge states are suppressed. The system switches only few times appropriately despite damage, active structural modes, and coupling nonlinearities.

\section{MMST-based Guaranteed Performance Adaptive Control (GPAC)}

One of the fundamental questions regarding the proposed damage-adaptive control scheme is that of guarantees that can be given that the proposed system will behave in the desired way under all possible values of $\theta$ and $x i$.

This is consistent with one of the main challenges in adaptive control, where an open issue is to derive suitable performance metrics for adaptive system, and develop strategies to assure that the dynamics of the overall adaptive system is close to that of a reference model for all time. If such performance metrics and control strategies could be found, then one could guarantee that both the transient and steady-state properties of the closed-loop system are close to that of the reference model. The most challenging issue here is that this needs to be achieved for any variation in plant parameters.

Here we present an initial analysis of the problem of achieving consistent system performance in adaptive control, and propose several solutions based on MMST.

Let the plant dynamics be described by:

$$
\dot{x}=f(p, x, u),
$$




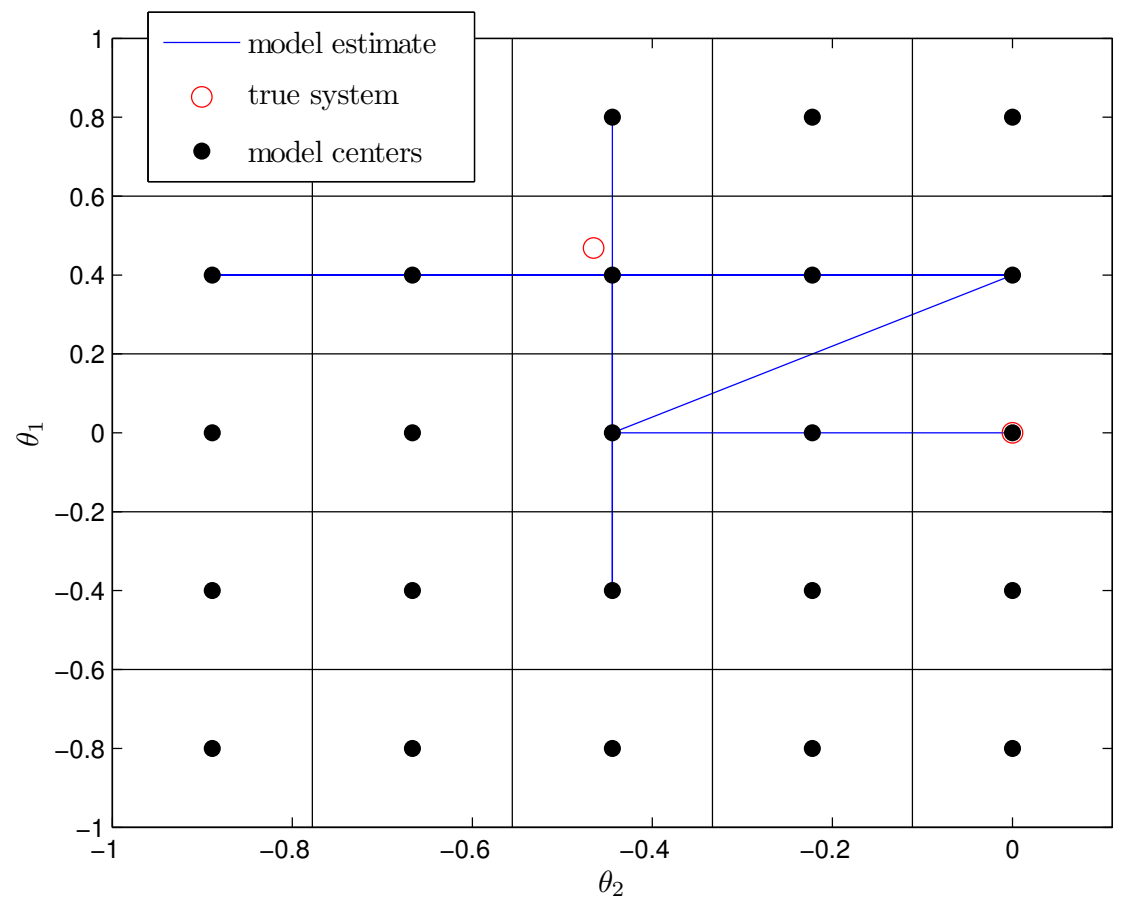

(a)
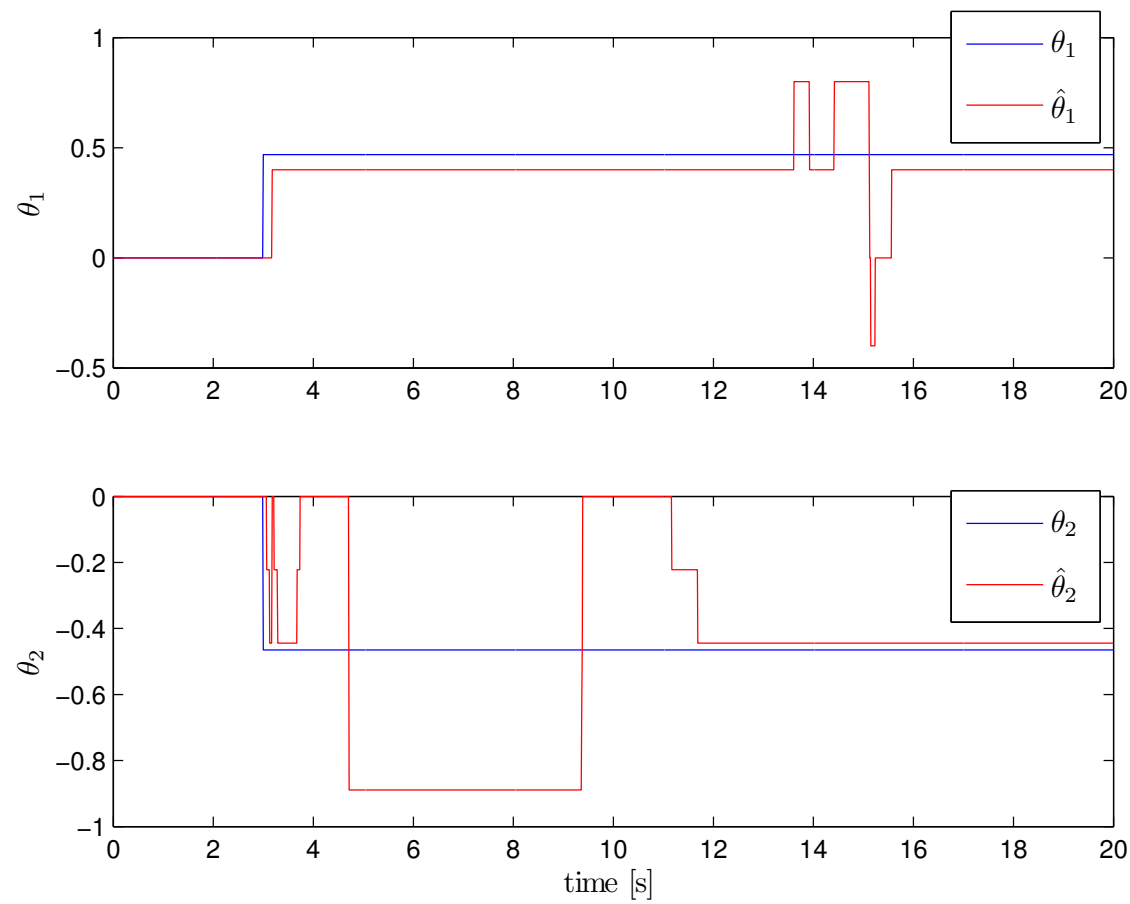

(b)

Figure 6. MMST with Fixed Models: Response of the estimates of the damage parameters under damage at $t=3$ seconds. 

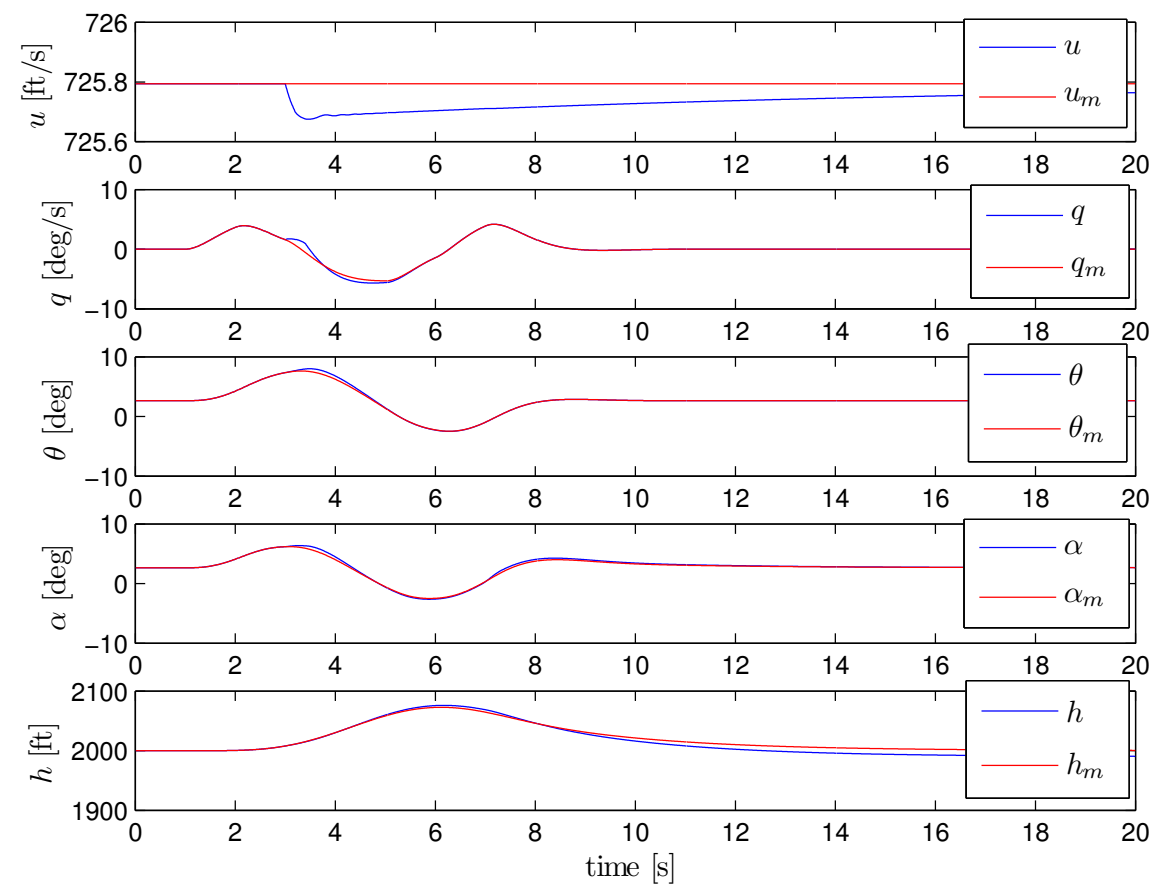

Figure 7. MMST with one Adaptive Model, Biasing and Decreased Selection Frequency: Response of the longitudinal states under damage at $t=3$ seconds.

where $x \in \mathbb{R} \rightarrow \mathbb{R}^{n}, u \in \mathbb{R} \rightarrow \mathbb{R}^{m}$, and $p \in \mathcal{S}_{p}$, where $\mathcal{S}_{p}$ is a known set.

Reference Model: Let a stable reference model be given as: $\dot{x}^{*}=g\left(x^{*}, r\right)$, where $r$ is a bounded piece-wise continuous reference input.

Adaptive Controller: Let $u(t)$ be an output of the adaptive controller defined as:

$$
u=h(x, r, \hat{p}),
$$

where $\hat{p}$ denotes an estimate of $p$ generated by:

$$
\dot{\hat{x}}=f(\hat{p}, x, u)+\Lambda(\hat{x}-x), \quad \dot{\hat{p}}=q(x, u, \hat{x}),
$$

and let $u^{*}=h\left(x^{*}, r, p\right)$.

We now consider the following definitions:

Definition 1 (Consistent Instantaneous Performance): The closed-loop system (27)-(29) achieves consistent instantaneous performance if and only if, for all $\left\|x_{o}\right\| \leq \delta_{x}$, all $\left\|x_{o}^{*}\right\| \leq \delta_{x}$, all $p \in \mathcal{S}_{p}$, and given $\delta_{x}>0$ and $\delta_{u}>0$,

$$
\left\|x(t)-x^{*}(t)\right\| \leq \delta_{x}, \quad\left\|u(t)-u^{*}(t)\right\| \leq \delta_{u}, \quad \forall t \geq t_{o} .
$$

Definition 2 (Consistent Interval Performance): The closed-loop system (27)-(29) achieves consistent performance over an interval $\left[t_{o}, T\right]$ if and only if, for all $x_{o}$ and $x_{o}^{*}$ in some predefined sets of initial conditions, all $p \in \mathcal{S}_{p}$, and given $\epsilon_{x}$ and $\epsilon_{u}$,

$$
\int_{t_{o}}^{T}\left\|x(t)-x^{*}(t)\right\| d t \leq \epsilon_{x}, \quad \int_{t_{o}}^{T}\left\|u(t)-u^{*}(t)\right\| d t \leq \epsilon_{u} .
$$

The main challenge here is to design a control strategy that will keep the above measures at or below their prespecified bounds for any change in the plant parameters. This is a highly complex problem whose solution depends on many factors. Some possible solutions are proposed below. 


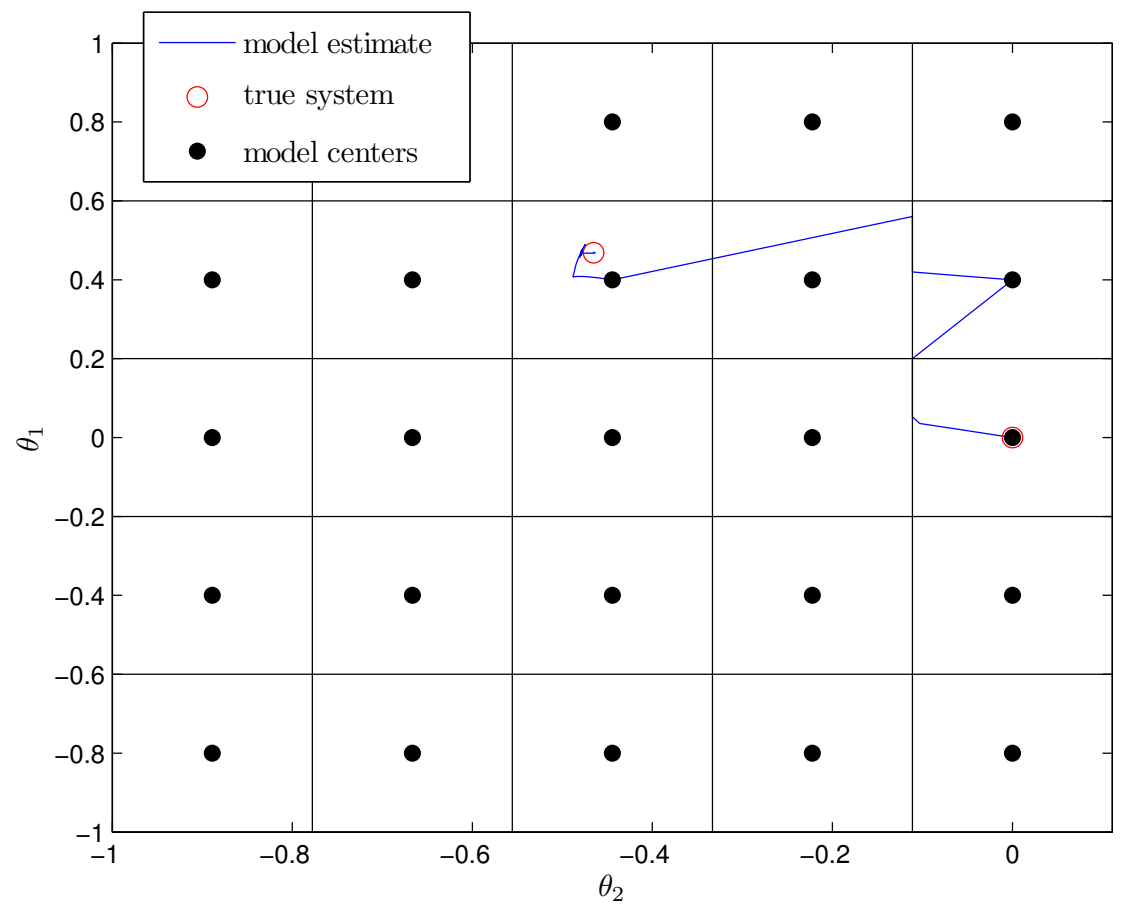

(a)
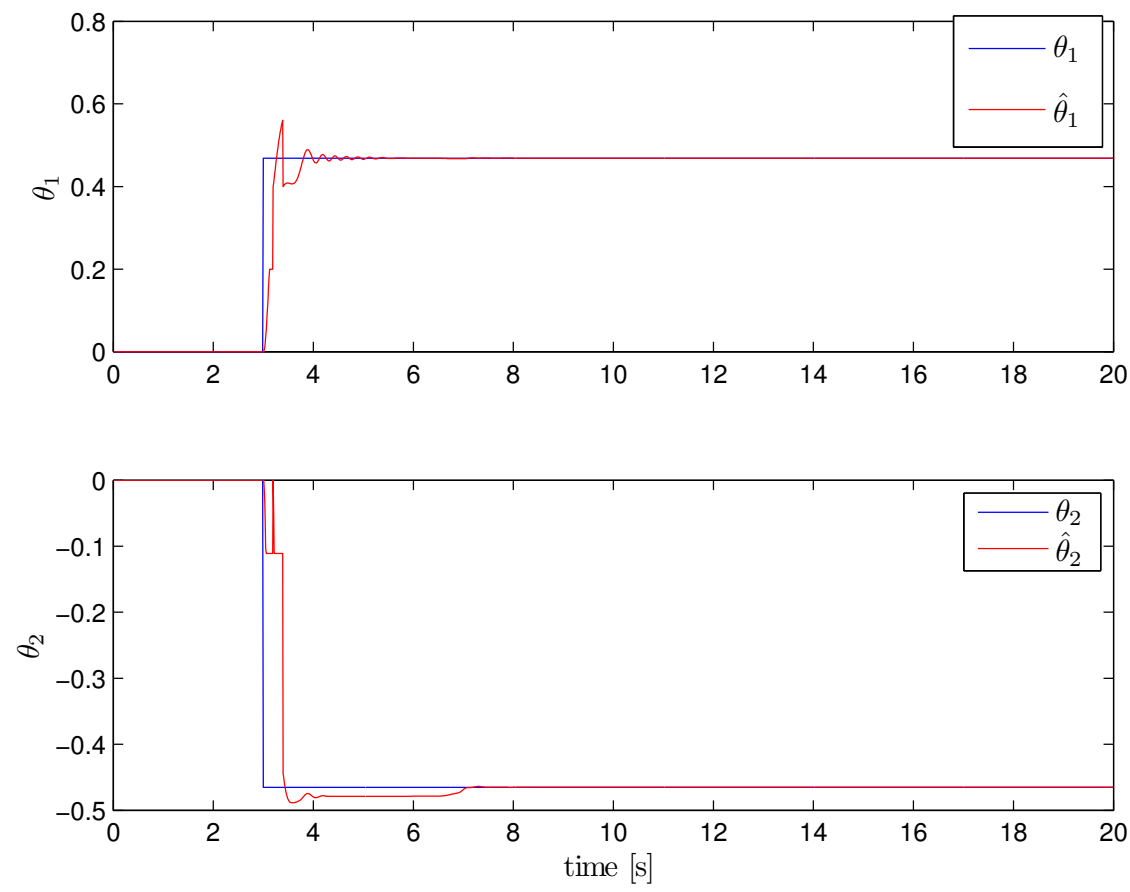

(b)

Figure 8. MMST with one Adaptive Model, Biasing and Decreased Selection Frequency: Response of the damage parameter estimates under damage at $t=3$ seconds. 

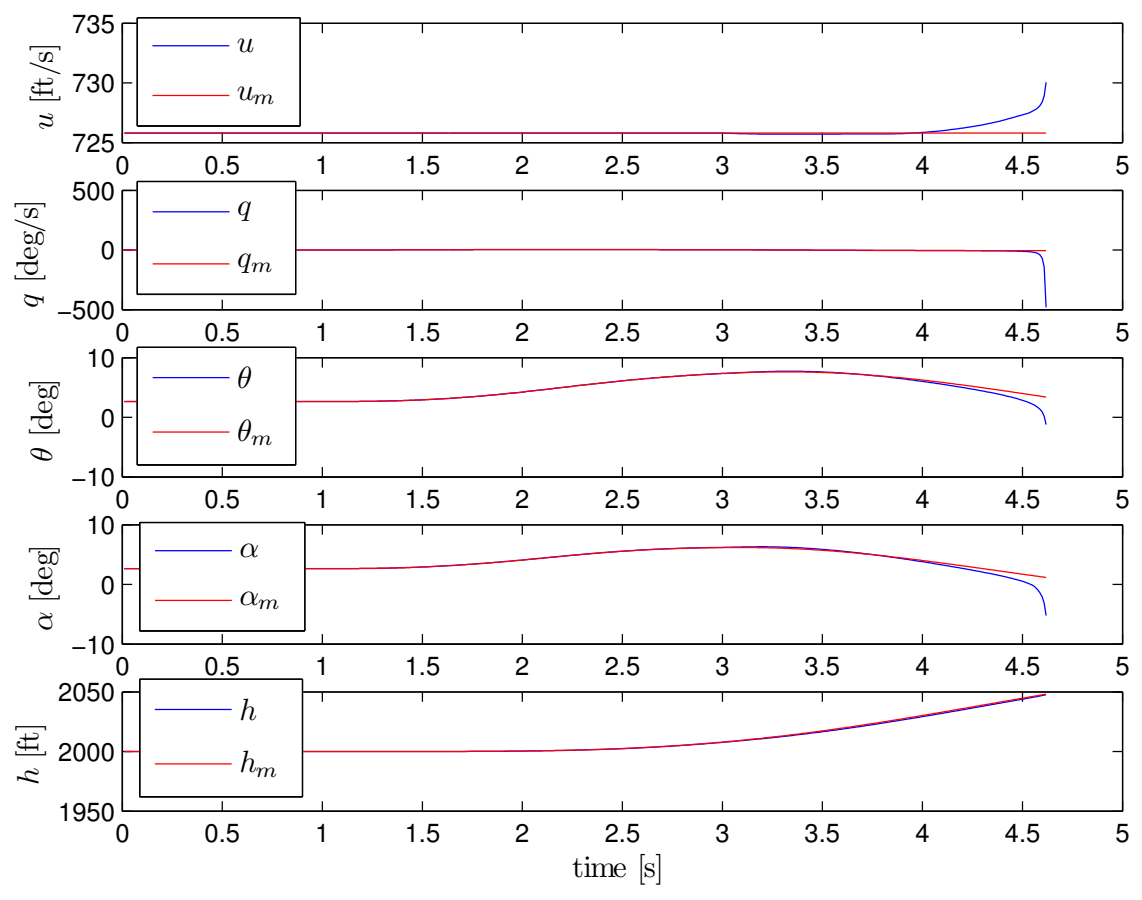

Figure 9. Response of the longitudinal states in the case of damage with coupling terms included, and no compensation.
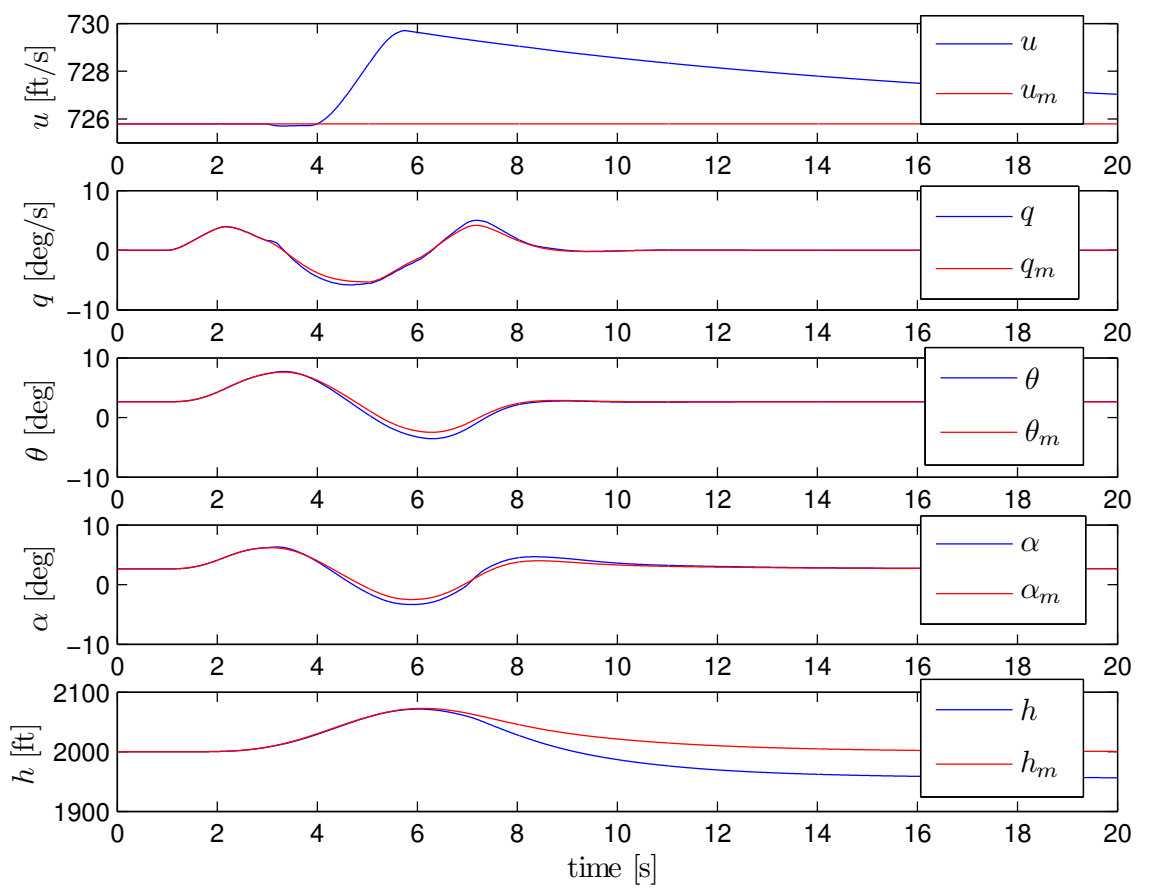

Figure 10. Response of the longitudinal states with coupling terms included and variable structure terms used to compensate for nonlinearities. 


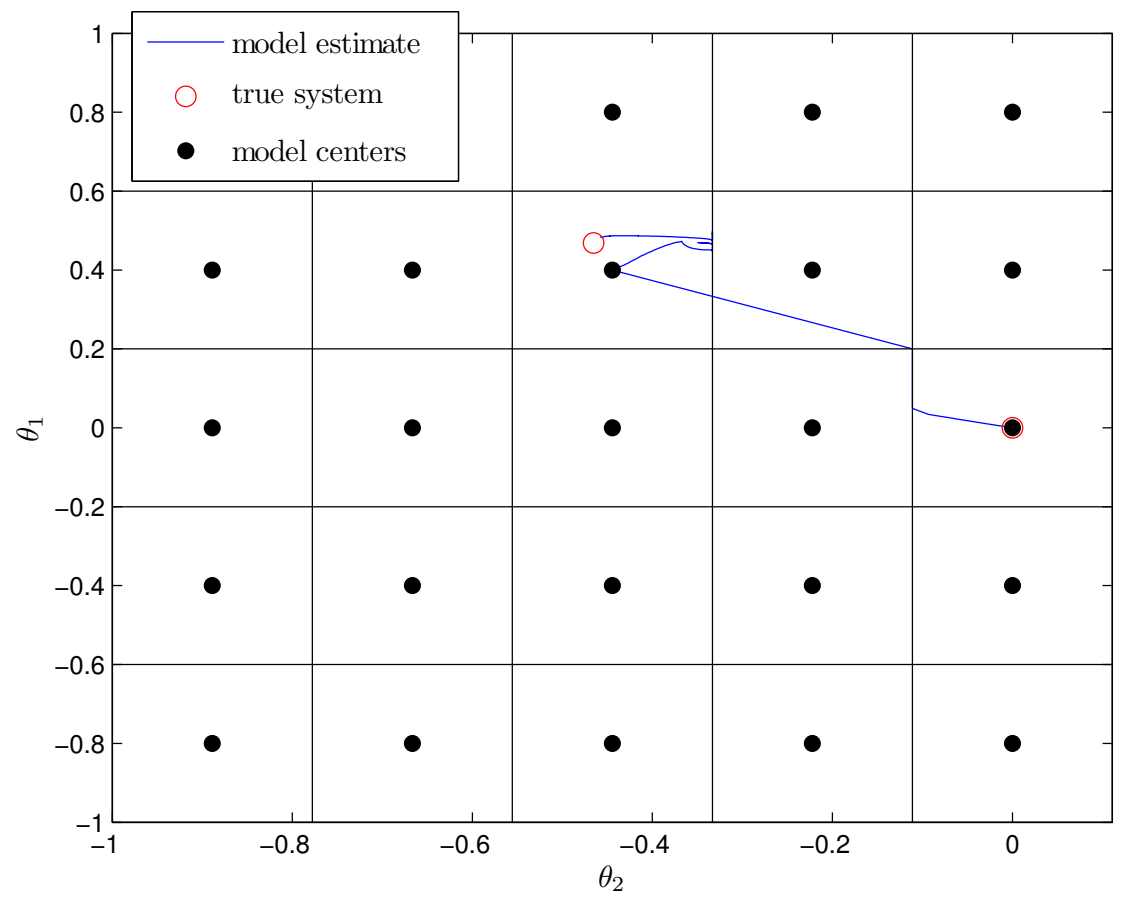

(a)
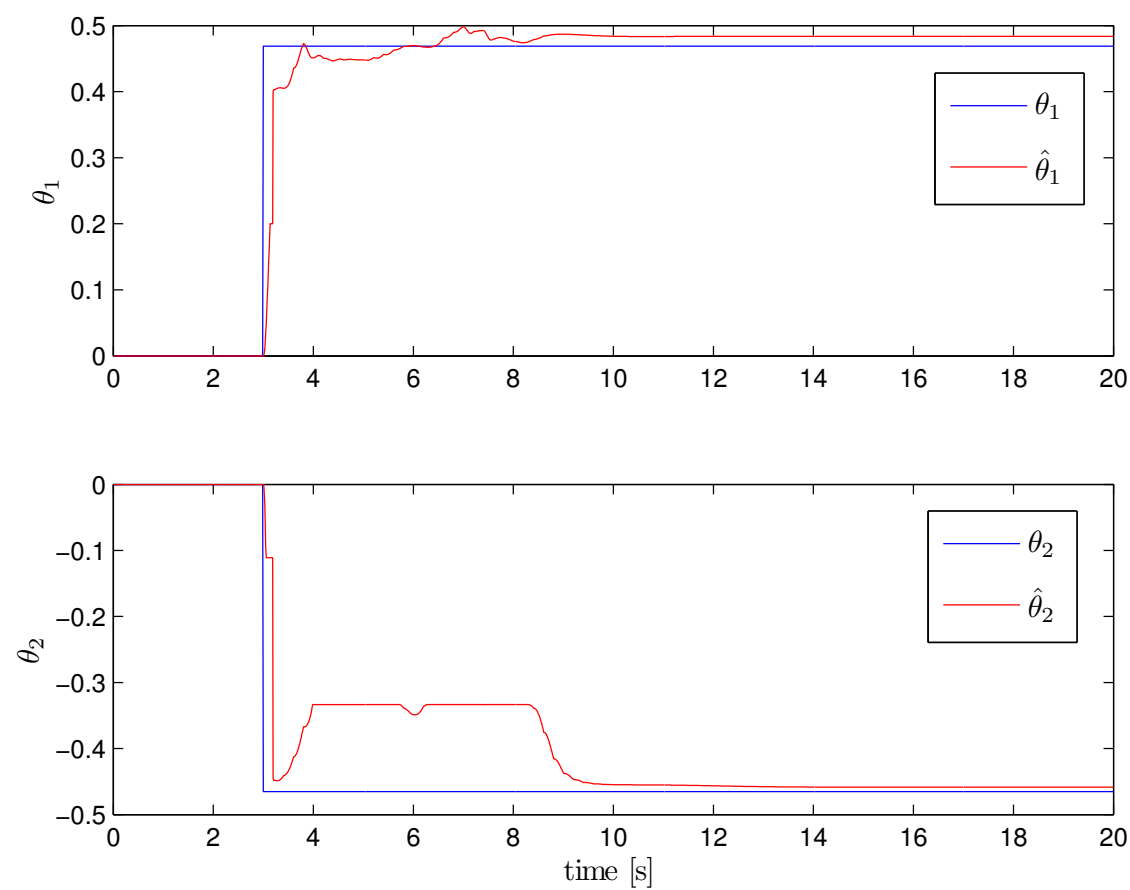

(b)

Figure 11. Response of the damage parameters and their estimates with variable structure terms used to compensate for nonlinearities. 

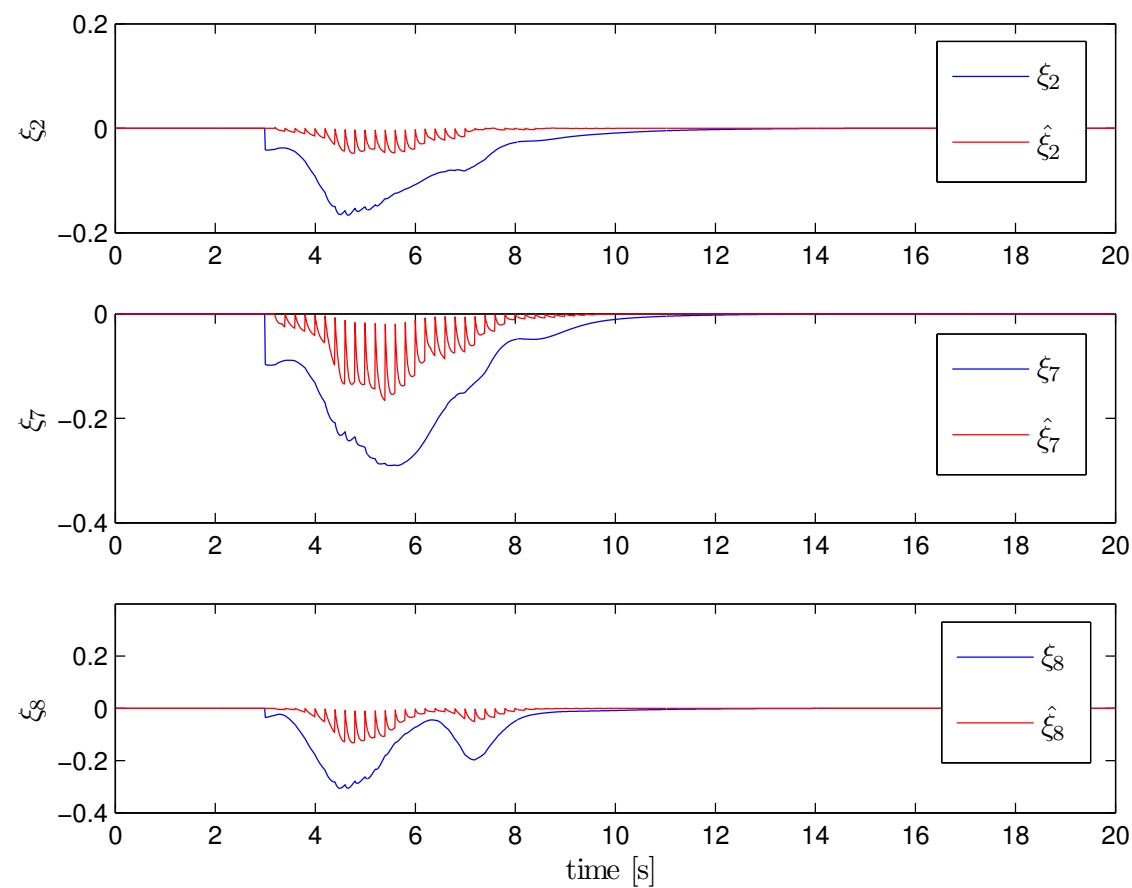

Figure 12. Response of the coupling terms and their estimates with variable structure terms used to compensate for nonlinearities.
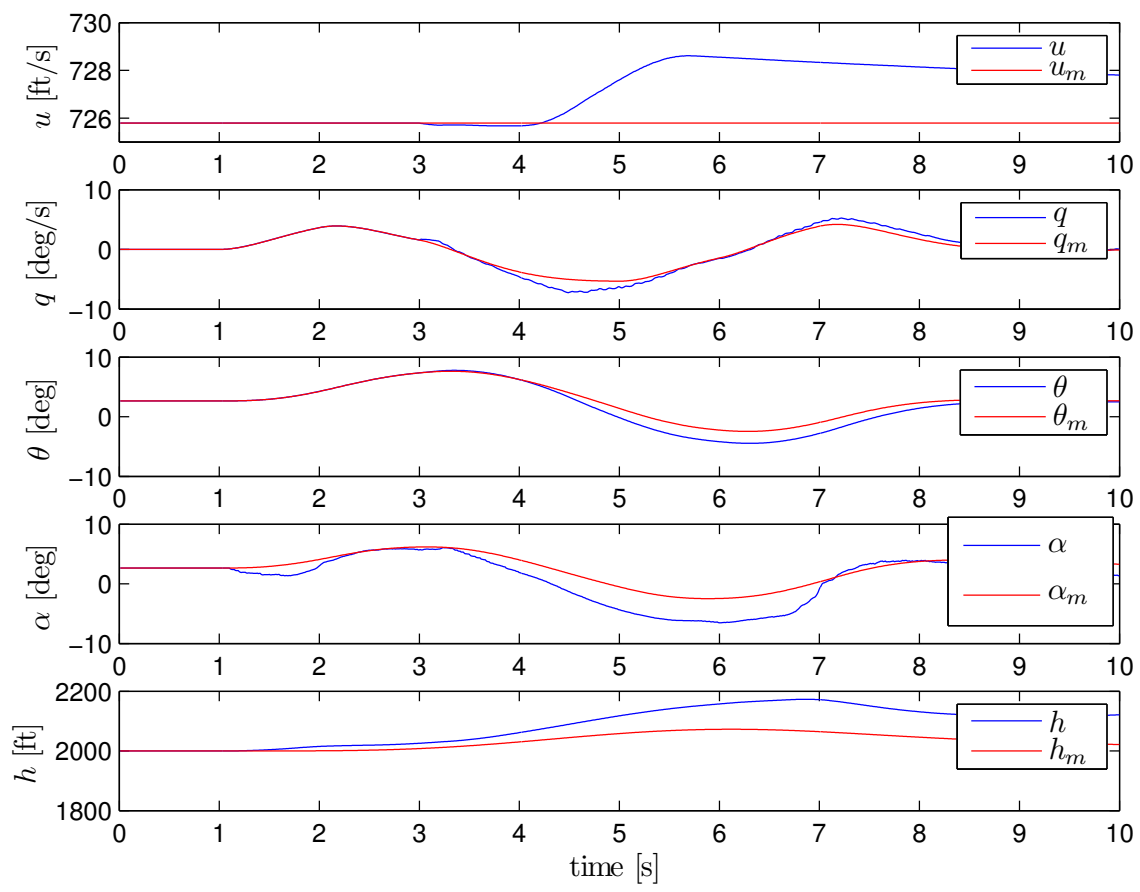

Figure 13. State response of the ASE model to damage at $t=3$ seconds in the case without compensation. 


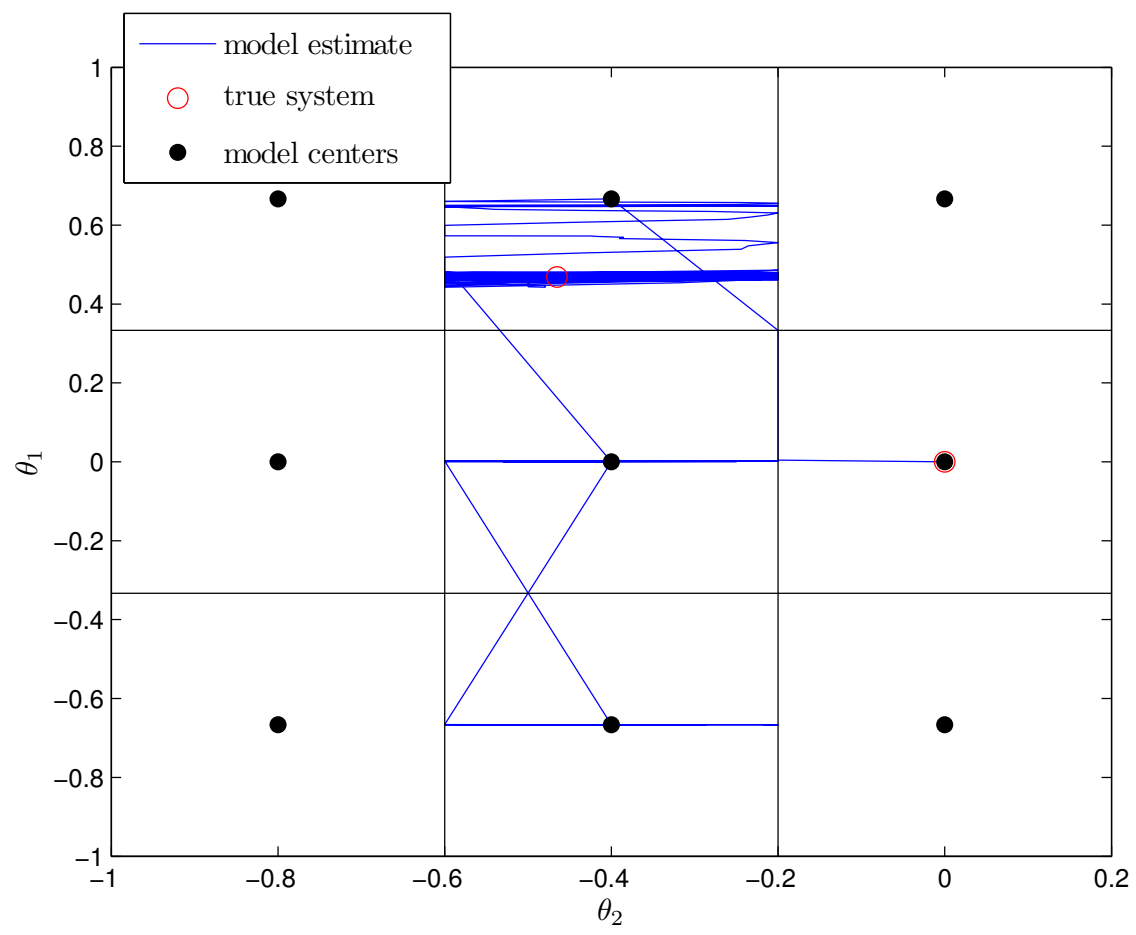

(a)
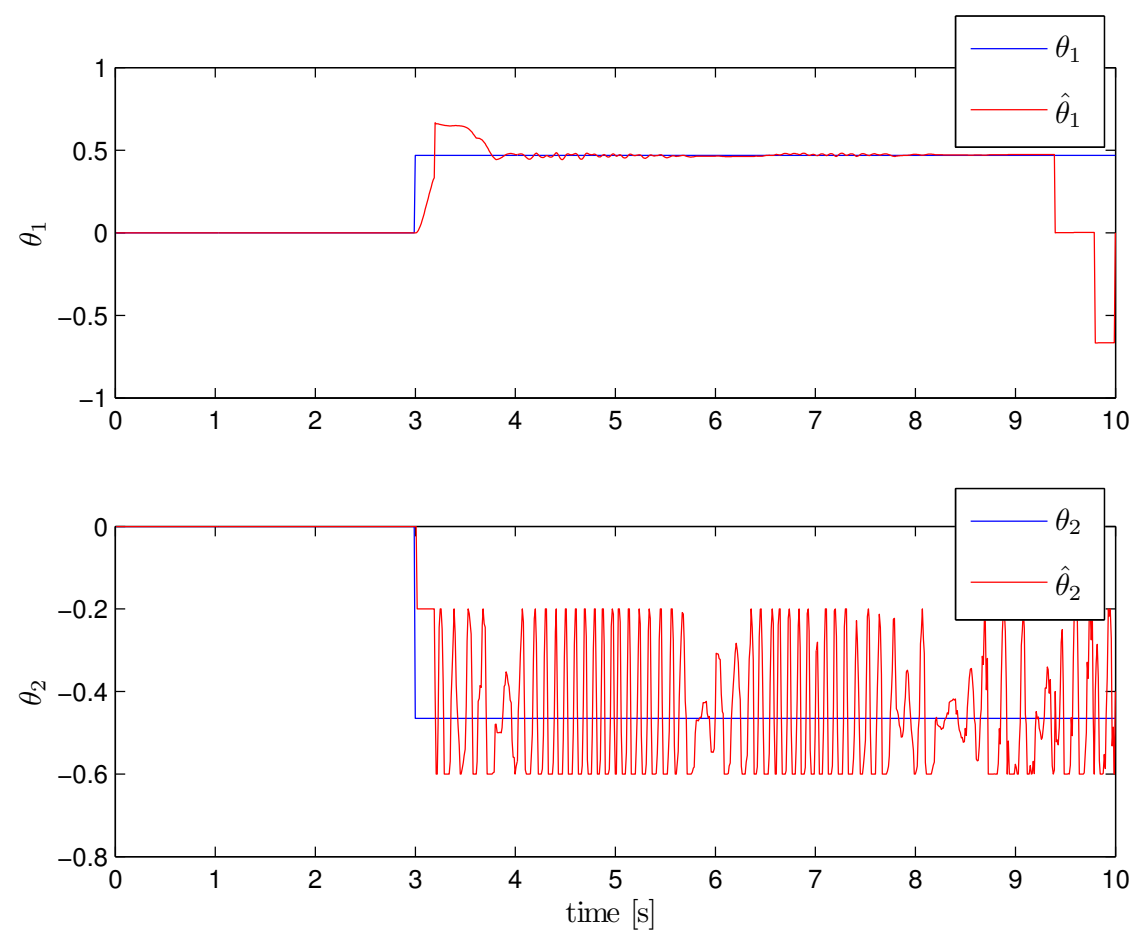

(b)

Figure 14. Response of the damage parameters and their estimates with no compensation for structural dynamics. 

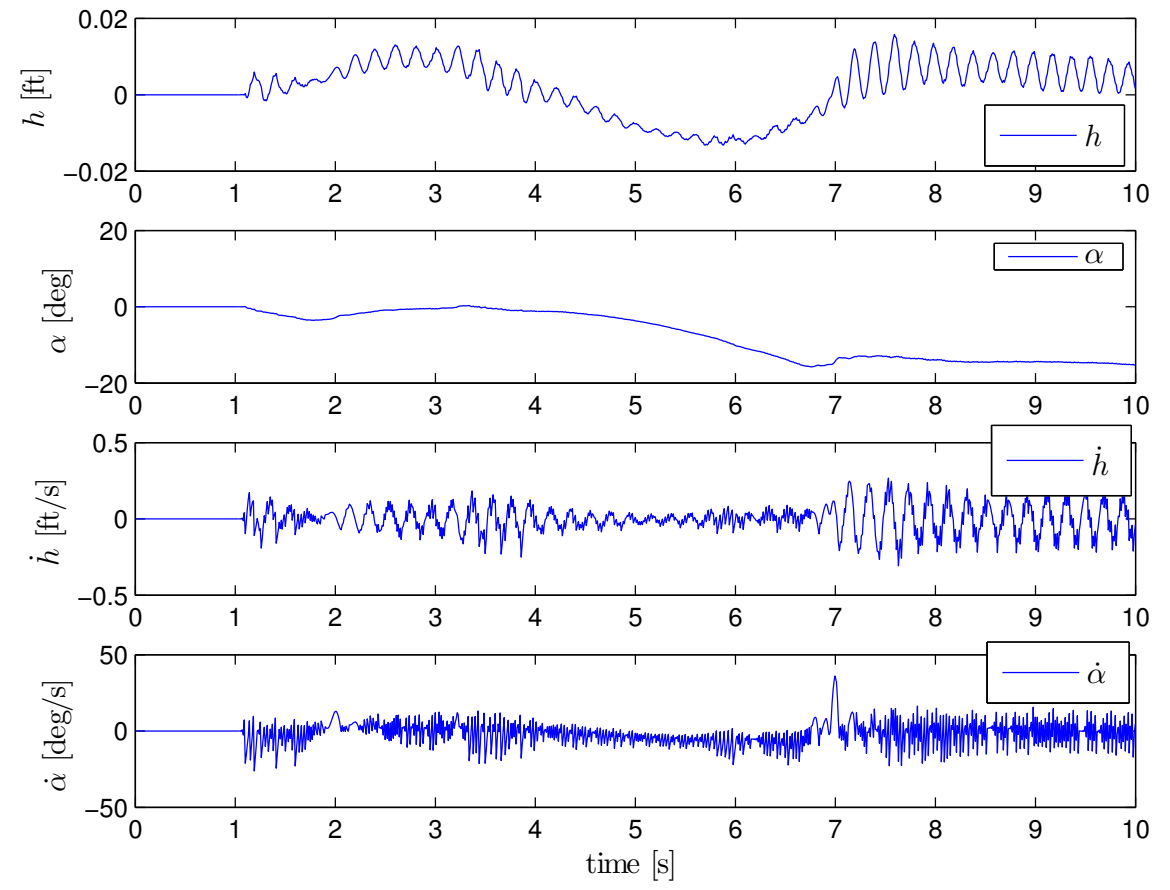

Figure 15. Response of the pitch-and-plunge states to damage at $t=3$ seconds in the case without compensation.
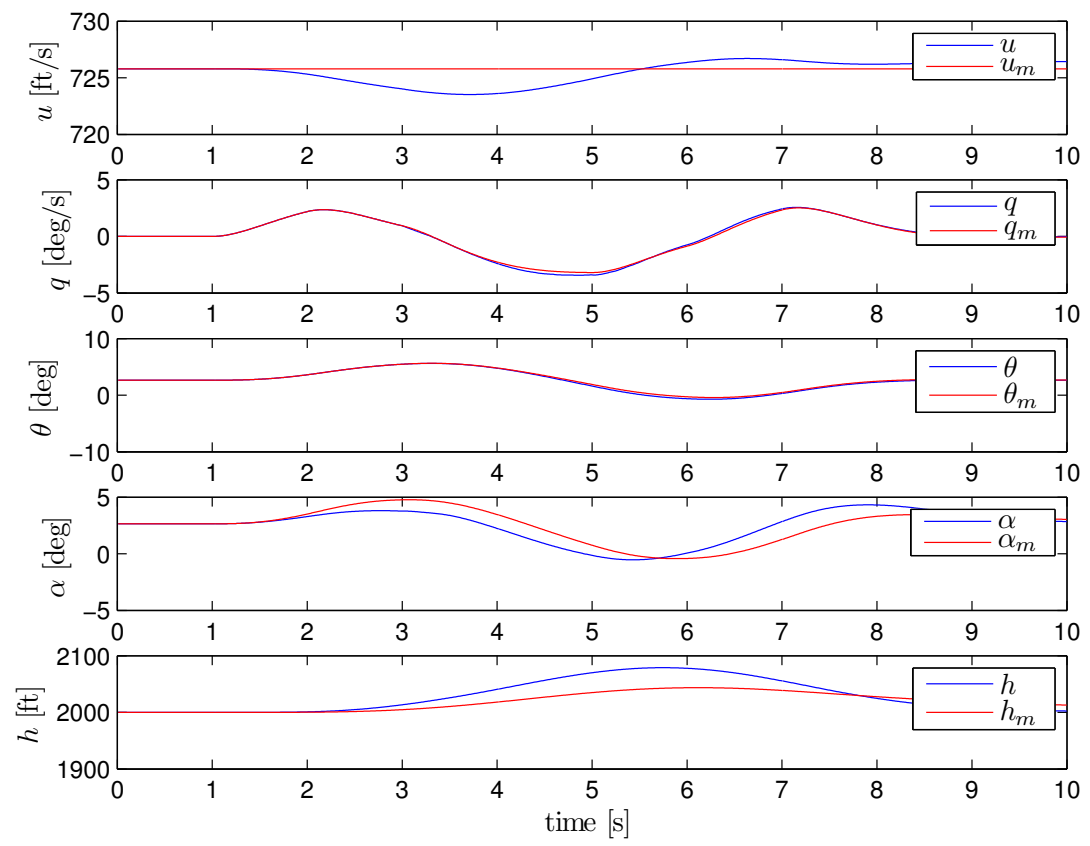

Figure 16. State response of the ASE model to damage at $t=3$ seconds in the case with compensation.

18 of 25 


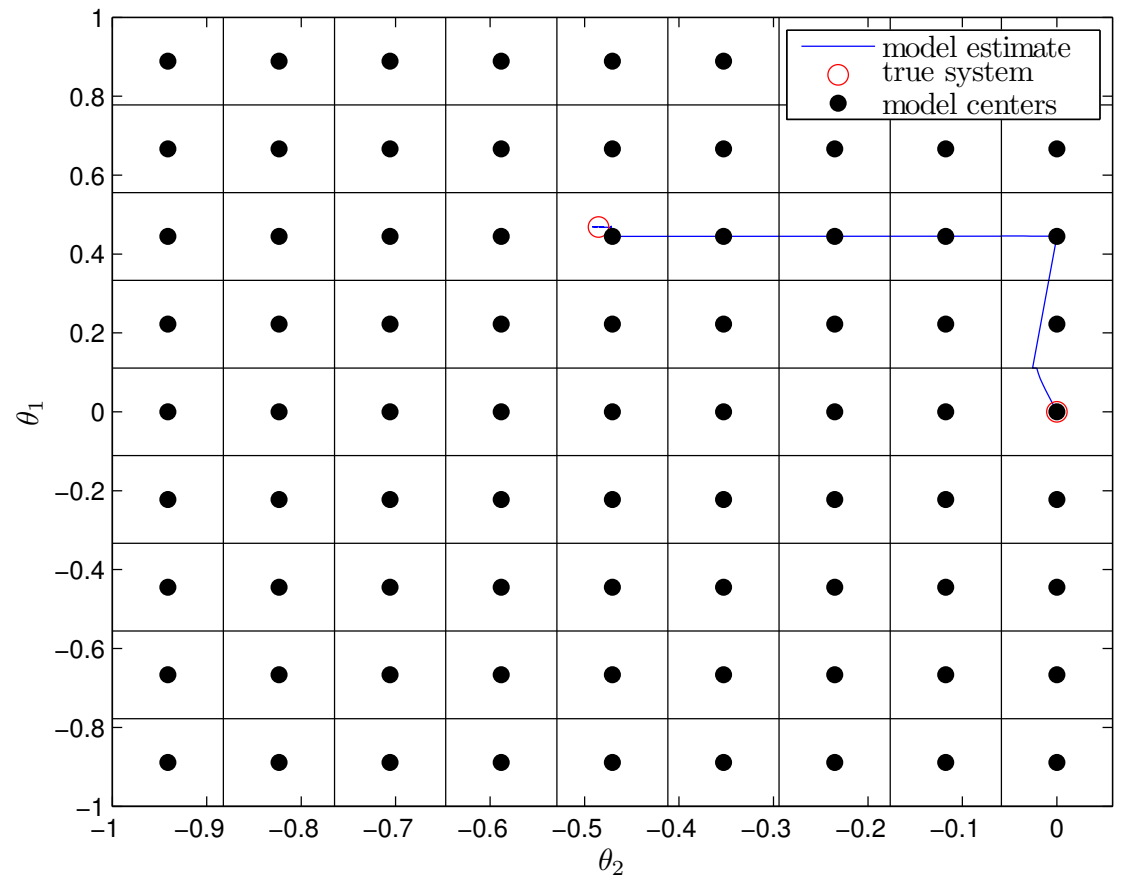

(a)
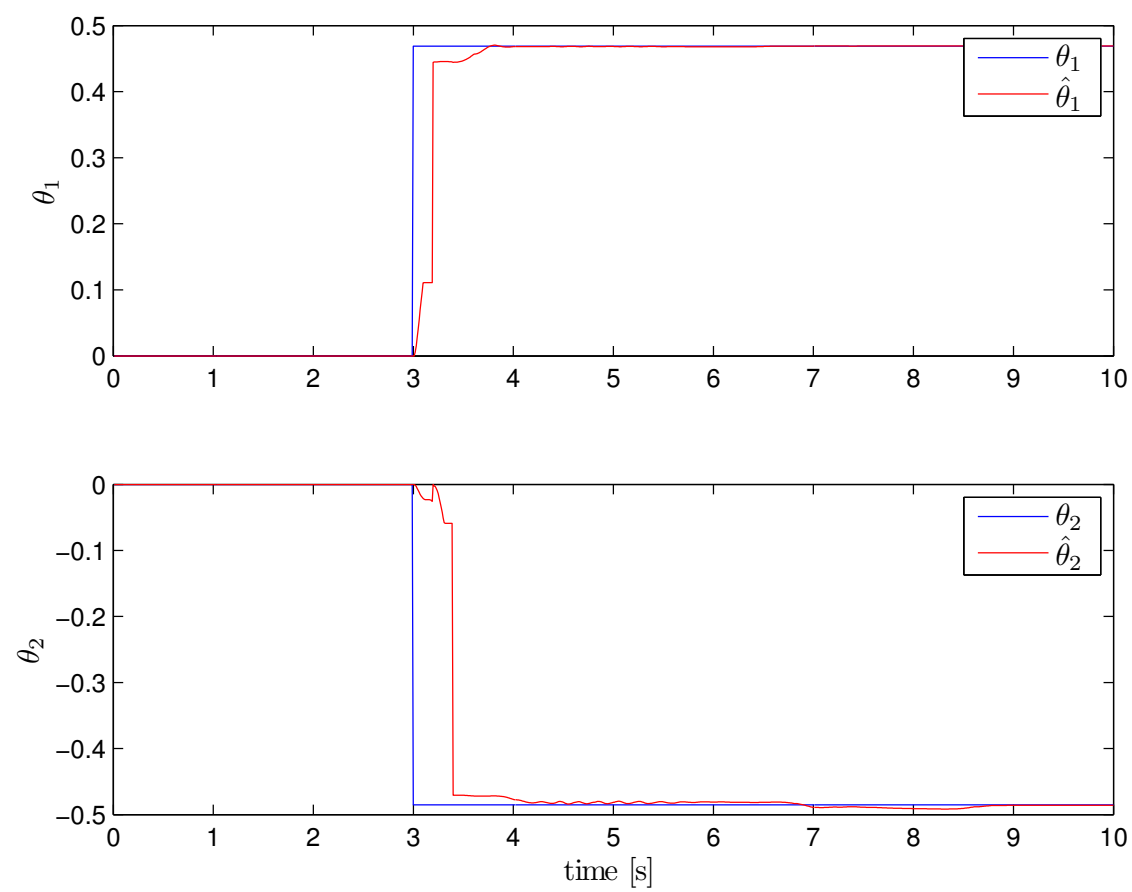

(b)

Figure 17. Response of the damage parameters and their estimates with compensation for structural dynamics. 

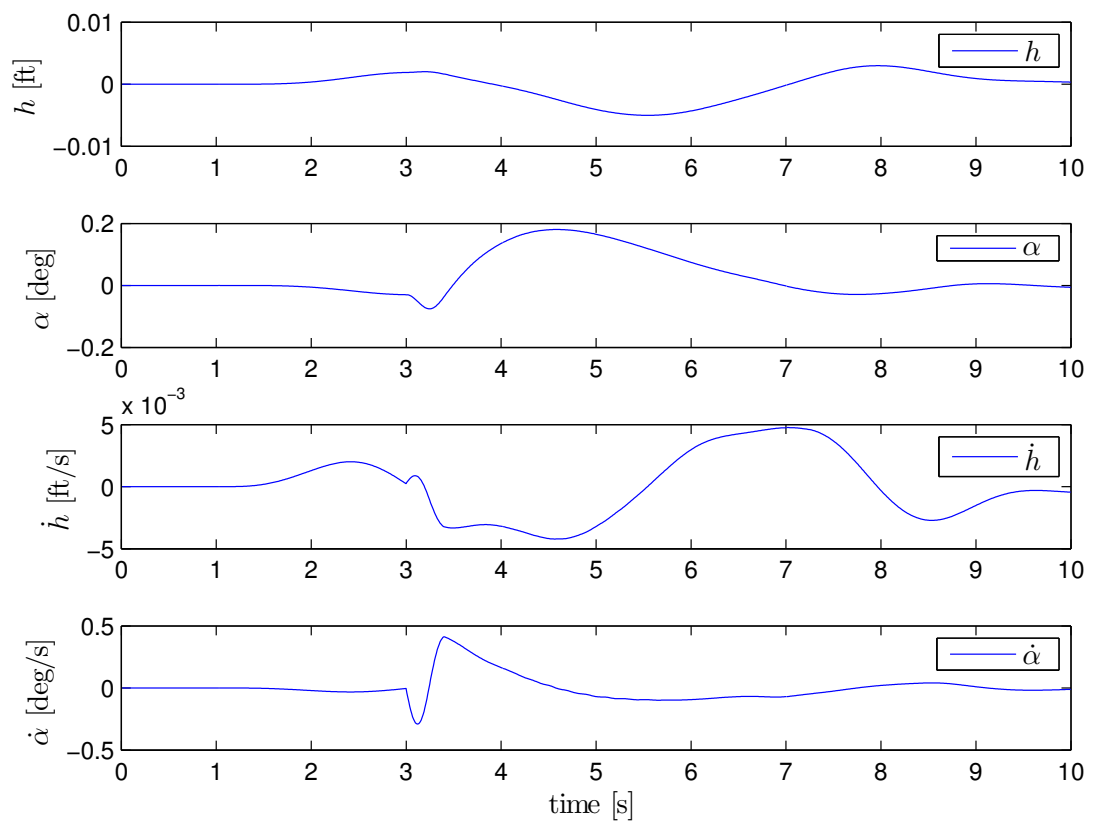

Figure 18. Response of the pitch-and-plunge structural states to damage at $t=3$ seconds in the case with compensation.

MMST with Model Injection: In this case the idea is to use all fixed observers and populate the subset to which the system switches with a large number of observers, Figure 19. This way at least one of the observers will be fairly close to the true plant dynamics. If the system adapts from there, the tracking error can be minimized along with the Consistent Performance performance index.

Simulation of a first-order plant with an uncertain control derivative coefficient is used to evaluate the modelinjection technique, and the resulting values of the Consistent Performance Index (CPI) against the level of uncertainty (from 0.05 to 1) is shown in Figure 20. It is seen that the model injection technique has a potential to minimize the CPI for any value of the uncertain parameter.

MMST with Adaptive Gain Scheduling: In this case all the observers are adaptive but the parameter estimates are adjusted using different adaptive gains, and the system will switch to the observer with the adaptive gain that is "the best" for the current operating regime. This approach is illustrated in Figure 21.

The same first-order example is used to illustrate the adaptive gain scheduling approach, and the results are shown in Figure 22. It is seen that the values of CPI are similar to those obtained with model injection. Hence this is another potentially feasible approach to CPI minimization.

MMST design using Model Sets: This strategy is based on the use of an output error feedback (OEF) term in the control law, Figure 23, and robust stability results to stabilize all plants within a subset of the parametric set. As shown recently, ${ }^{1}$ the resulting closed-loop adaptive control system is linear and time-varying. This OEF design procedure can be used for the Model Set Design, which addresses the question as to how many models should be used, and where they should be placed within the parametric set. The proposed approach is based on extending the current set until the robust stability conditions are violated, and designing a controller with output error feedback for the next subset. An important aspect of this approach is that, when the system switches to the right set, the resulting closed-loop system will have some minimum performance specified during the model-set design. For this reason this approach is also referred to as the Guaranteed Performance Models Set Design (GP-MSD). Simulation results using this strategy are shown in Figure 24 resulting in performance similar to that obtained in the previous cases. These strategies will be compared to find the one most suitable for minimizing the CPI in the case of multivariable plants with multiple uncertain parameters.

Guaranteed switching strategies: One of the major questions in MMST design is that of convergence of the switching parameter to the value closest to or corresponding to the actual plant dynamics. Many theoretical issues need to be addressed before one can derive analytic conditions for a finite-time interval over which the system is guaranteed 

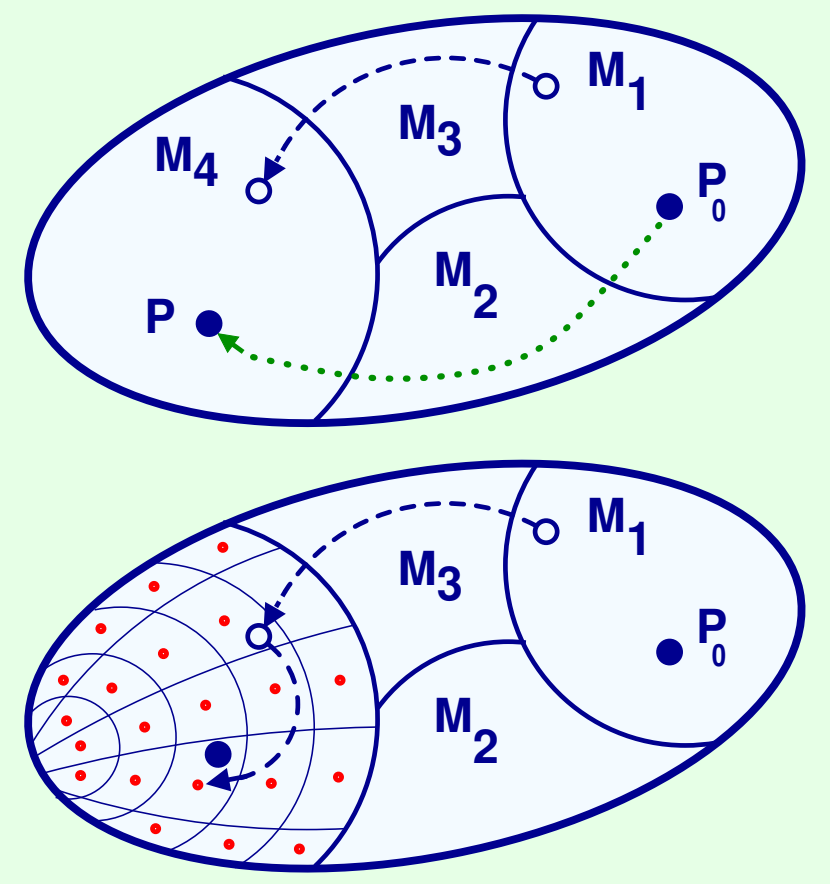

Figure 19. Strategy with model injection: The system will first switch to a fixed observer $M_{4}$ closest to the current plant dynamics. At that instant the set corresponding to $M_{4}$ is is populated with multiple observers. Adaptation is hence replaced by rapid switching to the observer closest to the current plant dynamics.

to switch to the "right" model. These include: performance criterion used for switching; model set design; overall switching strategy; adaptive control law; speed of parameter variations in the system; and signal excitation.

This aspect of the MMST design is extremely important since switching to the right model in finite time guarantees some minimum performance. Theoretical and practical aspects of this strategy need to be investigated in detail.

\section{Conclusions}

In this paper we present a comprehensive approach to damage accommodation using Model Set Design, MMST, and Variable Structure compensation for coupling nonlinearities.

In the paper a new approach is described related to the design of model sets for the MMST, given the bounds on the plant parameters. The MMST controller also includes terms that compensate for cross coupling nonlinearities arising due to the damage.

We also implemented the MMST approach to an integrated rigid-body and structural dynamics model that combines linearized F/A-18 dynamics with the pitch-and-plunge dynamics of the TAMU wing.

In all cases excellent performance was obtained despite damage, coupling nonlinearities, and structural dynamics. This demonstrates the feasibility of the MMST approach for damage accommodation.

While the augmented ASE model used in this paper is simple and based on ad-hoc assumptions, our further work will include testing of the IDACS controller on more realistic models such as the Generic Transport Model (GTM) simulation that accounts for changes in aerodynamics due to damage, as well as for changes in the center of gravity, retrofitted with an aeroservoelastic model.

\section{References}

${ }^{1}$ J. D. Bošković, N. Knoebel and R. K. Mehra "An Initial Study of a Combined Robust and Adaptive Control Approach to Guaranteed Performance Flight Control", to be presented at the 2008 American Control Conference, Seattle, WA, June 11-13, 2008. 


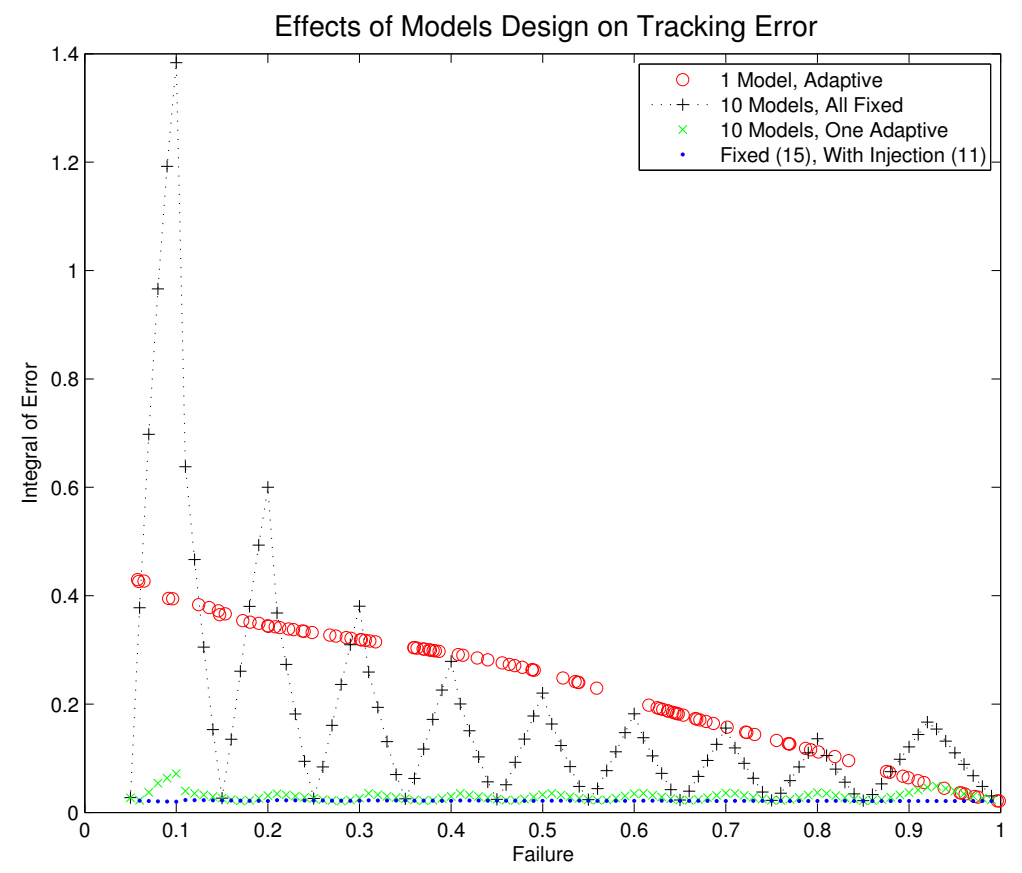

Figure 20. Comparison of the strategy with model injection with the following strategies: all-fixed observers, single adaptive observer, and all-fixed plus one adaptive observer.

${ }^{2}$ J. D. Bošković and R. K. Mehra, "Robust Integrated Flight Control Design Under Failures, Damage and State-Dependent Disturbances", AIAA Journal of Guidance, Control \& Dynamics, Vol. 28, No. 5, pp. 902-917, September-October 2005.

${ }^{3}$ J. D. Bošković, S. E. Bergstrom, R. K. Mehra, James Urnes, Sr., Mark Hood, and Yohan Lin, "Fast on-Line Actuator Reconfiguration Enabling (FLARE) System", Proceedings of the 2005 AIAA Guidance, Navigation and Control Conference, San Francisco, CA, August 15-18, 2005.

${ }^{4}$ J. D. Bošković, S. Bergstrom and R. K. Mehra, ”A Multi-Layer Intelligent Loss-of-Control Prevention System (LPS) for Flight Control Applications", Proceedings of the 2005 AIAA Guidance, Navigation and Control Conference, San Francisco, CA, August 15-18, 2005.

${ }^{5}$ J. D. Bošković and R. K. Mehra, "A Multiple Model Adaptive Flight Control Scheme for Accommodation of Actuator Failures", AIAA Journal of Guidance, Control E Dynamics, Vol 25, No. 4, pp. 712-724, July-August, 2002.

${ }^{6}$ J. D. Bošković and R. K. Mehra, 'Intelligent Adaptive Control of a Tailless Advanced Fighter Aircraft under Wing Damage", AIAA Journal of Guidance, Control E Dynamics, Vol. 23, No. 5, pp. 876-884, September-October 2000

${ }^{7}$ M. Brenner, "Aeroservoelastic Modeling and Validation of a Thrust-Vectoring F/A-18 Aircraft", NASA Technical Paper 3647, Sept. 1996.

${ }^{8}$ J. Brinker and K. Wise, "Reconfigurable Flight Control of a Tailless Advanced Fighter Aircraft", in Proceedings of the 1998 AIAA Guidance, Navigation and Control Conference, Vol. 1, pp. 75-87, Boston, MA, August 10-12, 1998

${ }^{9}$ Boeing Phantom Works, "Reconfigurable Systems for Tailless Fighter Aircraft - RESTORE (First Draft)", Contract No. F33615-96-C-3612, Scientific and Technical Reports, System Design Report, CDRL Sequence No. A007, St. Louis, Missouri, May 1998.

${ }^{10}$ M. Goman and A. Khrabrov, "State-Space Representation of Aerodynamic Characteristics of an Aircraft at High Angles of Attack", J. of Aircraft, Vol.31, No.5, 1994

${ }^{11} \mathrm{M}$. Goman et al. Mathematical description of aircraft's longitudinal aerodynamic characteristics at high angles of attack accounting for dynamic effects of separated flow. TsAGI Preprint.

${ }^{12}$ I. M. Gregory, "Dynamic inversion to control large flexible transport aircraft", AIAA-1998-4323, AIAA Guidance, Navigation, and Control Conf., Boston, 1998.

${ }^{13}$ I. M. Gregory, "Modified Dynamic Inversion to Control Large Flexible Aircraft - What's Going On?", AIAA-1999-3998, AIAA Guidance, Navigation, and Control Conf., Portland, 1999.

${ }^{14}$ I. M. Gregory, "Stability Result for Dynamic Inversion Devised to Control Large Flexible Aircraft", AIAA 2001-4284, AIAA Guidance, Navigation, and Control Conf., Montreal, 2001.

${ }^{15}$ Y. Miyazawa, "Flight Control Law and Structural Filter Design for a Flexible Aerospace Vehicle", AIAA-2000-4256, AIAA Guidance, Navigation, and Control Conf., Denver, 2000.

${ }^{16}$ M. Moshier, R. Hinrichsen and G. Czarnecki, "Exploring Better Ways to Determine In-Flight Wing Damage: Dynamic Loading Methodologies", Aircraft Survivability, pp. 21-26, Spring 2002.

${ }^{17}$ K. S. Narendra and J. Balakrishnan, "Adaptive Control using Multiple Models", IEEE Transactions on Automatic Control, Vol. 42, No. 2 , pp. 171-187, February 1997. 


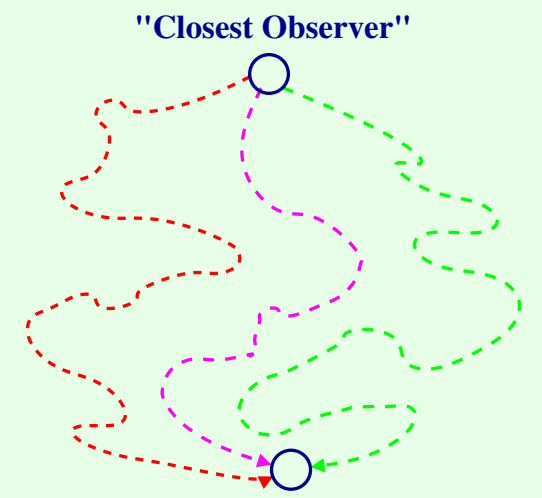

Current Plant Dynamics

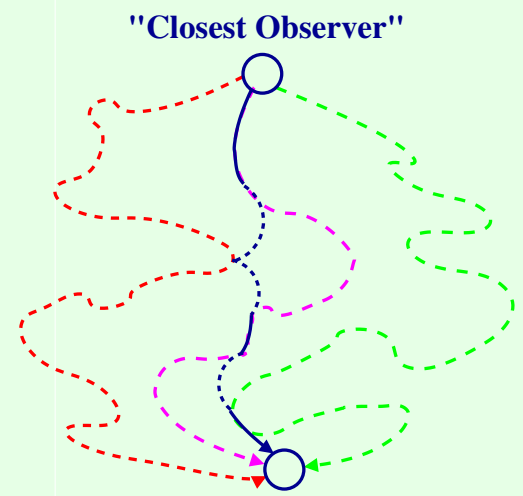

Current Plant Dynamics

Figure 21. Different adaptive gains will result in different system trajectories (left figure). The MMST approach will result in moving along a current trajectory (solid blue line) or switching from one trajectory to another (dashed blue line) to improve the response (right figure).

${ }^{18}$ N. Nguyen, K. Krishnakumar and J. Kaneshige, ”Dynamics and Adaptive Control for Stability Recovery of Damaged Asymmetric Aircraft”, Proceedings of the AIAA Guidance, Navigation, and Control Conference, August 21-24 2006, Keystone, Colorado.

${ }^{19}$ K. Wise, E. Lavretsky, J. Zimmerman, J. Francis, D. Dixon, and B. Whitehead, ”Adaptive Flight Control of a Sensor Guided Munition”, AIAA GNC Conference, San Fracisco, August 2005.

${ }^{20}$ G. Plantanitis, and T. Strganac, "Control of a Nonlinear Wing Section Using Leading- and Trailing-Edge Surfaces", Journal of Guidance, Control E Dynamics, Vol. 27, No. 1, pp. 52-58, January-February 2004. 


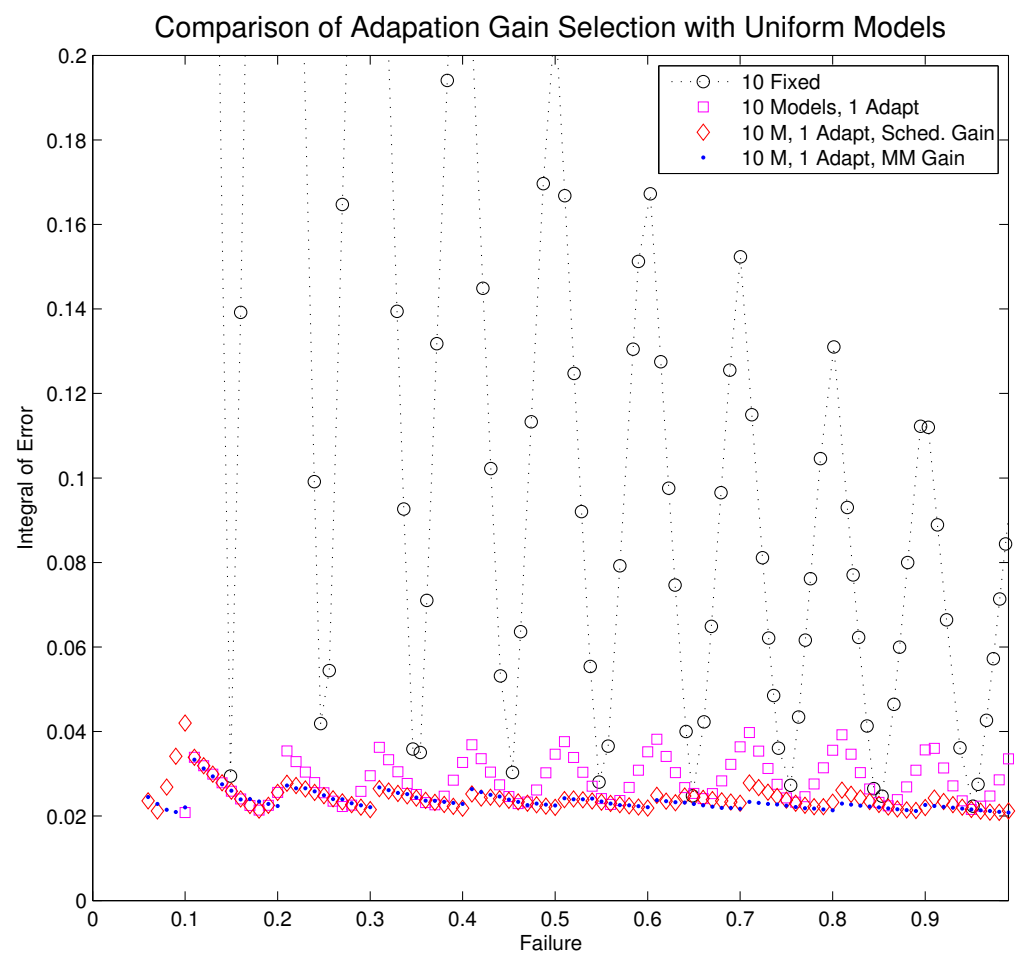

Figure 22. Comparison of the strategy with adaptive gain scheduling with the following strategies: all-fixed observers, all-fixed plus one adaptive observer, and all-fixed plus one adaptive observer with scheduled gains.

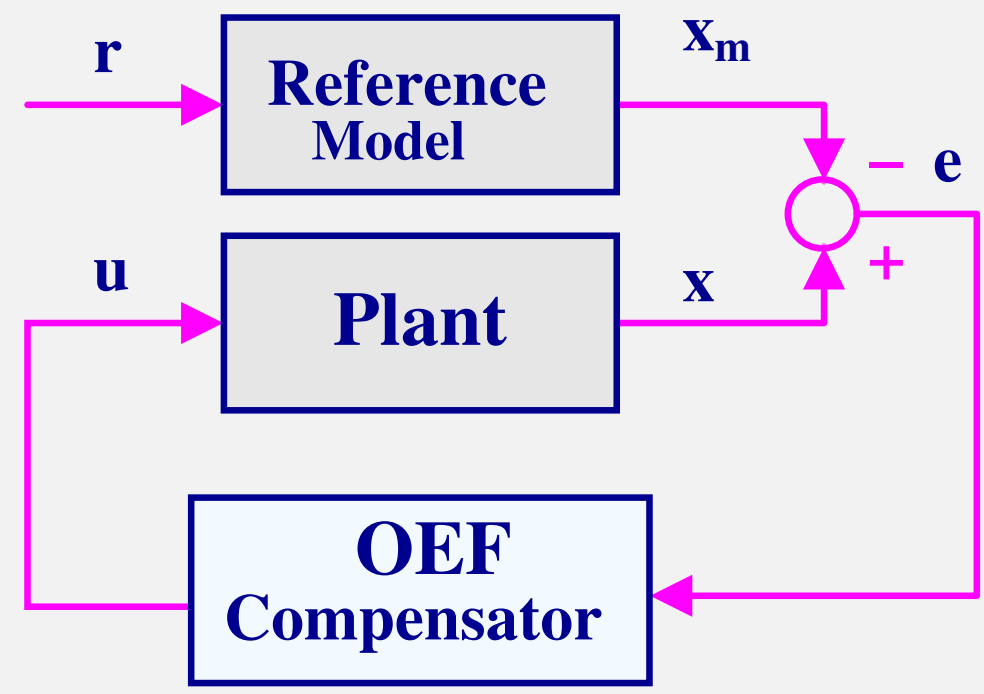

Figure 23. Structure of the system with output error feedback. 


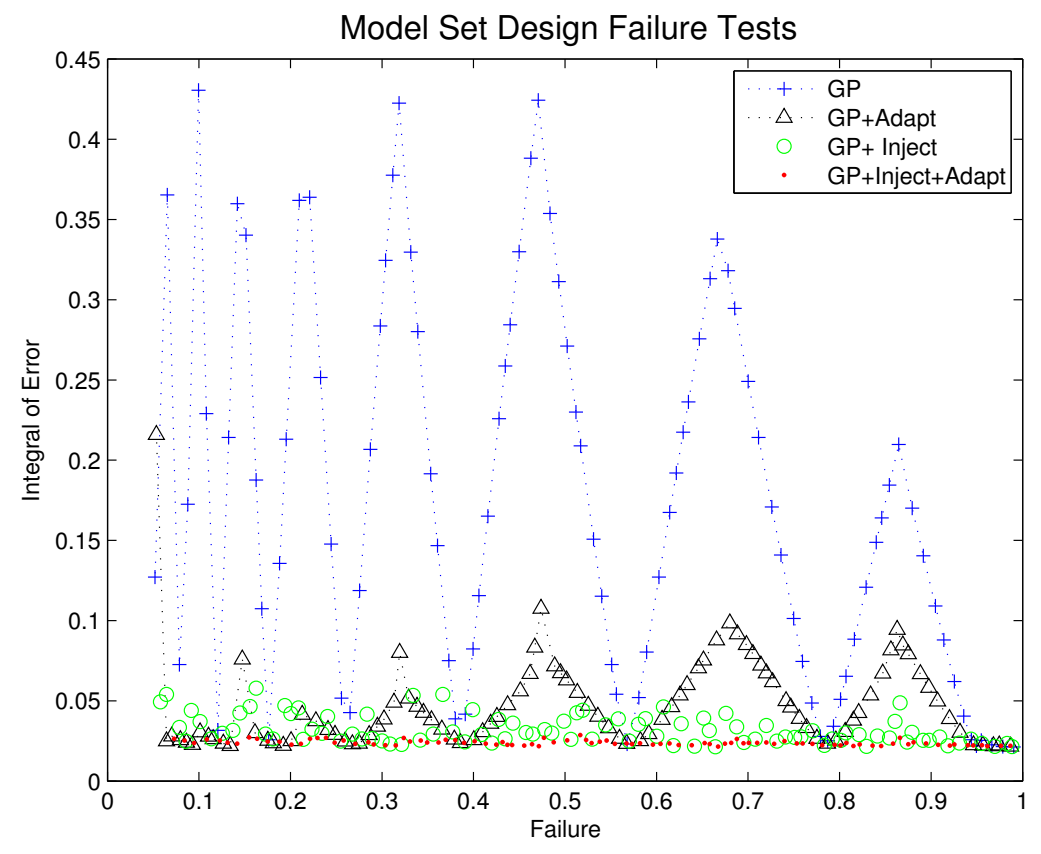

Figure 24. Comparison of the GP-MSD strategies. 